\title{
Long-Lasting Visuo-Vestibular Mismatch in Freely-Behaving Mice Reduces the Vestibulo- Ocular Reflex and Leads to Neural Changes in the Direct Vestibular Pathway
}

\author{
Julie Carcaud, ${ }^{1}$ Filipa França de Barros, ${ }^{1}$ DErwin Idoux, ${ }^{1}$ Daniel Eugène, ${ }^{1}$ Lionel Reveret, ${ }^{2}$ \\ Lee E. Moore, ${ }^{3}$ Pierre-Paul Vidal, ${ }^{3}$ and ${ }^{\circledR}$ Mathieu Beraneck ${ }^{1}$
}

\section{DOI:http://dx.doi.org/10.1523/ENEURO.0290-16.2017}

${ }^{1}$ Center for Neurophysics, Physiology, Pathology, CNRS UMR 8119, Université Paris Descartes, Sorbonne Paris Cité, Paris, France, ${ }^{2}$ INRIA Grenoble, Rhône-Alpes, Laboratoire Jean Kuntzmann, UMR 5224, France, ${ }^{3}$ Cognition and Action Group, CNRS UMR 8257, Université Paris Descartes, Sorbonne Paris Cité, Paris, France

\begin{abstract}
Calibration of the vestibulo-ocular reflex (VOR) depends on the presence of visual feedback. However, the cellular mechanisms associated with VOR modifications at the level of the brainstem remain largely unknown. A new protocol was designed to expose freely behaving mice to a visuo-vestibular mismatch during a 2-week period. This protocol induced a 50\% reduction of the VOR. In vivo pharmacological experiments demonstrated that the VOR reduction depends on changes located outside the flocculus/paraflocculus complex. The cellular mechanisms associated with the VOR reduction were then studied in vitro on brainstem slices through a combination of vestibular afferent stimulation and patch-clamp recordings of central vestibular neurons. The evoked synaptic activity demonstrated that the efficacy of the synapses between vestibular afferents and central vestibular neurons was decreased. In addition, a long-term depression protocol failed to further decrease the synapse efficacy, suggesting that the VOR reduction might have occurred through depression-like mechanisms. Analysis of the intrinsic membrane properties of central vestibular neurons revealed that the synaptic changes were supplemented by a decrease in the spontaneous discharge and excitability of a subpopulation of neurons. Our results provide evidence that a long-lasting visuo-vestibular mismatch leads to changes in synaptic transmission and intrinsic properties of central vestibular neurons in the direct VOR pathway. Overall, these results open new avenues for future studies on visual and vestibular interactions conducted in vivo and in vitro.
\end{abstract}

Key words: multisensory; neuronal excitability; reflex; synaptic plasticity; vestibular neurons; VOR

\section{Significance Statement}

Calibration of the vestibulo-ocular reflex depends on the presence of visual feedback. In vivo work has suggested that cerebellar-dependent calibration of VOR is, in the long-term, consolidated in the brainstem. However, the associated cellular mechanisms remain unknown. To address these mechanisms, we present an innovative protocol in which freely behaving mice are submitted to $15 \mathrm{~d}$ of visuo-vestibular mismatch. We demonstrated that this protocol leads to a $50 \%$ reduction of the VOR. We also showed that in brainstem slices, long-term VOR reduction is associated with synaptic and intrinsic changes within the vestibular nuclei, in the direct VOR pathway. This study opens new avenues for future studies on visual and vestibular interactions conducted both in vivo and in vitro. 


\section{Introduction}

Because of its relative simplicity, precise quantitative methods, and ease in applying experimental perturbations, gaze stabilization represents a suitable model to study motor learning that occurs when visual or vestibular sensory signals are modified (Blazquez et al., 2004; Broussard et al., 2011). In rodents, gaze stabilization depends on two complementary reflexes: the optokinetic reflex (OKR) that produces eye movements in the direction of visual motion and the vestibulo-ocular reflex (VOR) that stabilizes gaze during head motion (Stahl, 2004). Both reflexes cooperate to stabilize the visual scene on the retina in response to movement (Angelaki, 2004; Cullen, 2012).

Although the VOR operates even in the dark, its calibration depends on the presence of visual feedback. When a mismatch of visual and vestibular information occurs, retinal slip results, which blurs vision. This retinal slip serves as an error signal that is conveyed to the inferior olive and then to the cerebellum, where it is integrated with other sensory inputs (Fig. 1). The error signal indicates that eye movements are not compensatory, and thus drives motor learning to modulate the VOR (Shin et al., 2014) and restore gaze stabilization, a process known as VOR adaptation. The role of the cerebellum in the induction and short-term retention of this motor learning is clearly established (Hansel et al., 2001; Boyden et al., 2004; Carey, 2011; De Zeeuw and Ten Brinke, 2015). In contrast, long-term retention of the memory in the cerebellum itself or its transfer to downstream structures has long been a topic of debate (Broussard and Kassardjian, 2004; Broussard et al., 2011). One hypothesis was that the cerebellum is the main site of memory retention, as demonstrated by long-term depression at the synapses between parallel fibers and Purkinje cells

Received September 26, 2016; accepted January 10, 2017; First published January 16, 2017

The authors declare no competing financial interests.

Author contributions: J.C. and M.B. designed research; J.C., F.F.D.B., E.I., D.E., L.R., and M.B. performed research; J.C., F.F.D.B., E.I., L.R., L.E.M., and M.B. analyzed data; J.C., F.F.D.B., E.I., D.E., L.E.M., P.-P.V., and M.B. wrote the paper.

This research was supported by the Centre National de la Recherche Scientifique and the University Paris Descartes. El and MB receive support from the Centre National des Etudes Spatiales. JC and MB receive support from the French ANR-13-CESA-0005-02. FFB and MB receive support from the French ANR-15-CE32-0007.

Acknowledgments: We thank L. McElvain and S. Du Lac for their advice on the methodology for the vestibular afferents stimulation. We are grateful to $P$. Jegouzo for his technical support; this work would have not been possible without his innovative thinking in designing the WVM device. We thank M. Tagliabue for his help in developing the in vivo mouse experiments. We also thank C. Adou for her technical support for in vitro experiments. We thank J. McIntyre and J.X. Brooks for critically reading the manuscript.

Correspondence should be addressed to M. Beraneck. Center for Neurophysics, Physiology, Pathology, CNRS UMR 8119, 45 rue des St-Pères 75270, Université Paris Descartes, Paris, France. E-mail: mathieu.beraneck@ parisdescartes.fr.

DOI:http://dx.doi.org/10.1523/ENEURO.0290-16.2017

Copyright (C) 2017 Carcaud et al.

This is an open-access article distributed under the terms of the Creative Commons Attribution 4.0 International, which permits unrestricted use, distribution and reproduction in any medium provided that the original work is properly attributed.
(Fig. 1; Marr, 1969; Albus, 1971; Ito, 1982). An alternative model proposed that the cerebellum would not be the only site of retention but would also provide a teaching signal guiding the induction of plasticity within the brainstem (Miles and Lisberger, 1981; Lisberger et al., 1984). In favor of this hypothesis, experiments using cerebellum deactivation demonstrated that flocculi shutdown suppresses VOR short-term, but not long-term, adaptation (Luebke and Robinson, 1994; Pastor et al., 1994; Nagao and Kitazawa, 2003; Kassardjian et al., 2005). The retention of oculomotor memories outside the cerebellum in the long-term was further confirmed by OKR experiments (Shutoh et al., 2006; Anzai et al., 2010; Okamoto et al., 2011).

Although the hypothesis of a consolidation of long-term VOR changes in the brainstem has received support from many theoretical studies (Porrill and Dean, 2007; Masuda and Amari, 2008; Menzies et al., 2010; Medina, 2011; Clopath et al., 2014; Yamazaki et al., 2015), it has little experimental support. Studies in vivo have shown that some vestibular nuclei neurons changed their activity after VOR adaptation (Keller and Precht, 1979; Lisberger, 1988; Lisberger et al., 1994), even though this effect could not be dissociated from Purkinje cell activity. It was also proposed that the modification of the strength of the synapse between vestibular afferents and central vestibular neurons could be a key mechanism involved in VOR calibration (Menzies et al., 2010; Yamazaki et al., 2015).

Many of the studies on VOR motor learning have been conducted on animal models that do not allow for in vitro investigation. On the other hand, the use of the mouse model has its own constraints, as long-term modification of the VOR is classically achieved through passive headfixed, iterated discontinuous training sessions interrupted by intertrial intervals of variable duration (Boyden and Raymond, 2003). Here, the neural basis of VOR plasticity was evaluated with a new long-term VOR reduction procedure in mice. Using a combination of behavioral analyses, oculomotor measurements with or without floccular deactivation, and in vitro electrophysiological recordings, we provide evidence that long-term VOR reduction is accompanied by synaptic and intrinsic modifications in the direct VOR pathway.

\section{Material and Methods}

\section{Animals and surgical procedures}

All animal procedures were performed in accordance with the University Paris Descartes animal care committee's regulations. A total of $116 \mathrm{C} 57 \mathrm{BL} / 6 \mathrm{~J}$ male mice (Janvier Labs; RRID: IMSR_JAX:000664) aged 6-8 weeks were included in the protocol. Gas anesthesia was induced using isoflurane. The head was shaved using an electric razor, and a 2-cm longitudinal incision of the skin was made to expose the skull. A small custom-built head holder $(0.3 \times 0.3 \times 0.5 \mathrm{~mm})$ was fixed to the skull just anterior to the lambda landmark using dental cement (C\&B Metabond; Parkell). After the surgery, animals were isolated and closely monitored for $48 \mathrm{~h}$. Buprenorphine $(0.05 \mathrm{mg} / \mathrm{kg})$ was provided for postoperative analgesia, 


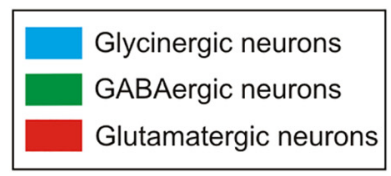

\section{VOR circuitry}

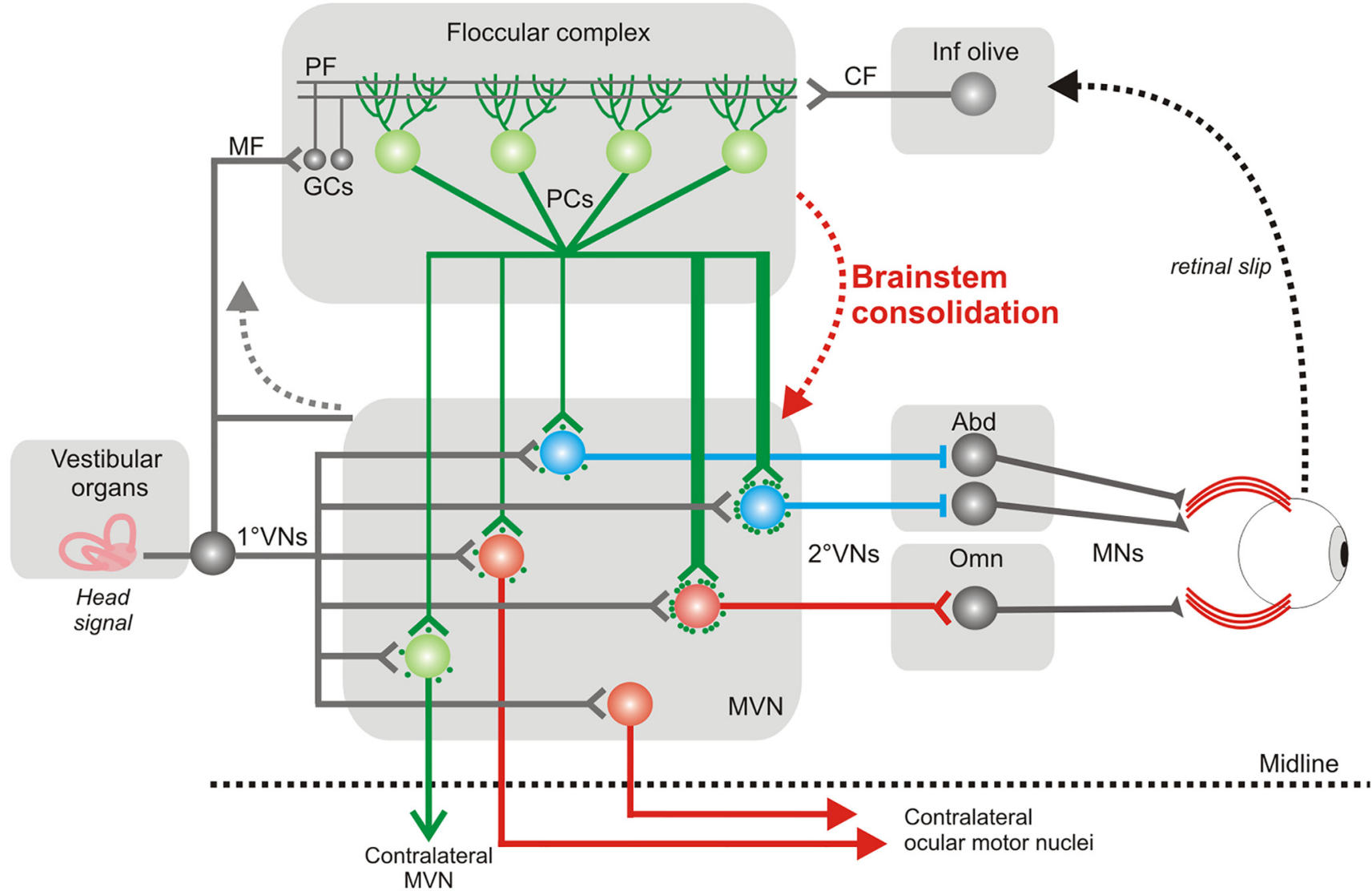

Fig. 1. Circuitry of structures implicated in VOR and its calibration. Integration of vestibular and visual inputs in the floccular complex modulates PC outputs. Floccular target neurons in the MVN are partitioned depending on the density of innervations received from the flocculi (thin or thick lines; density of synaptic contacts), on their neurotransmitter content, and projection sites. $1^{\circ} \mathrm{VN}$ and $2^{\circ} \mathrm{VN}$, first- and second-order vestibular neuron; MN, motoneuron; CF, MF, and PF, climbing, mossy, and parallel fiber; GC, granule cell; Abd and Omn, abducens and oculomotor nucleus.

and care was taken to avoid hypothermia and dehydration.

\section{Visuo-vestibular mismatch protocol}

Two days after the surgery, a custom-built device was secured on top of the head holder. The device consisted of a helmet $(2.2 \mathrm{~cm}$ width $\times 1.5 \mathrm{~cm}$ depth $\times 1.5 \mathrm{~cm}$ length; weight $2 \mathrm{~g}$ ) that completely covered the mouse's head. The front of the device was adapted to the mouse anatomy so that the nose was not covered, and its width allowed for grooming and barbering behaviors. To preserve light-dependent physiology and nychthemeral rhythm, the device was made of nonopaque plastic with a thickness of $0.3 \mathrm{~mm}$. In addition, 3-mm large vertical black stripes were drawn on the external surface to produce a high-contrast head-fixed visual signal during selfgenerated movements (Fig. $2 A$, top). A similar procedure was used on sham animals, except that the device was attached upside-down so that it did not cover the face of the mouse (Fig. $2 A$, bottom). Therefore, sham animals were exposed to the same surgical procedures and wore the same device but did not experience the visualvestibular mismatch. Visuo-vestibular mismatch (VVM) and sham animals were housed together. Animals were housed in groups of three to stimulate social interactions. After 2 weeks with the device on the head, mice were immediately tested in behavioral experiments or used for in vitro electrophysiological experiments. The VOR of mice used for electrophysiology measures was not recorded to avoid relearning/extinction processes.

\section{General observations}

During the first hours of the VVM protocol, mice displayed disturbed behavior including difficulties orienting in the cage, bumping into walls, and reduction in social interactions. Initial difficulties to access food and drink were also noted, and animals therefore received intensive attention during the initial $48 \mathrm{~h}$ of the protocol. This early period corresponded to a decrease in the weight of the animal (Fig. 2B). On the other hand, sham mice did not 
A

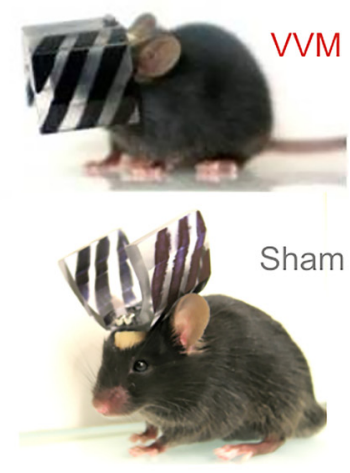

B

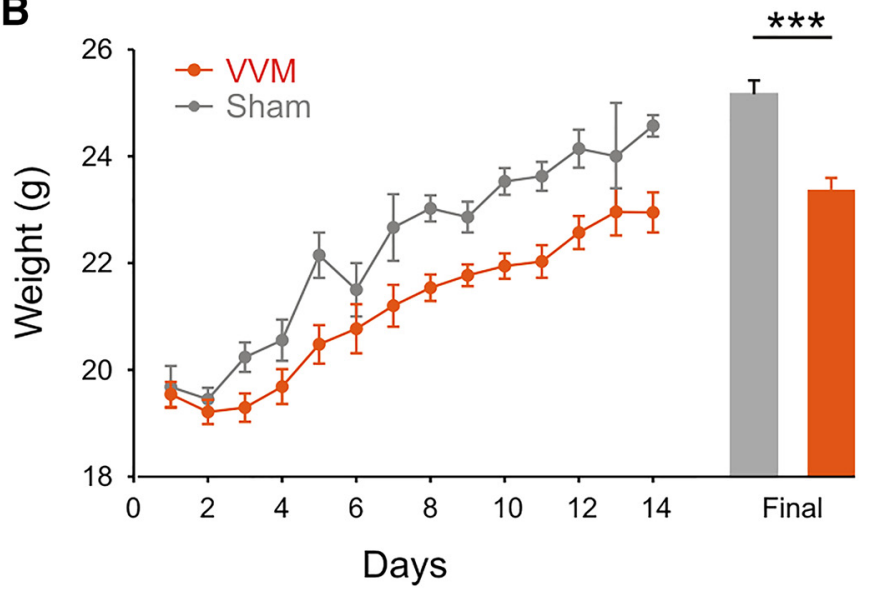

C
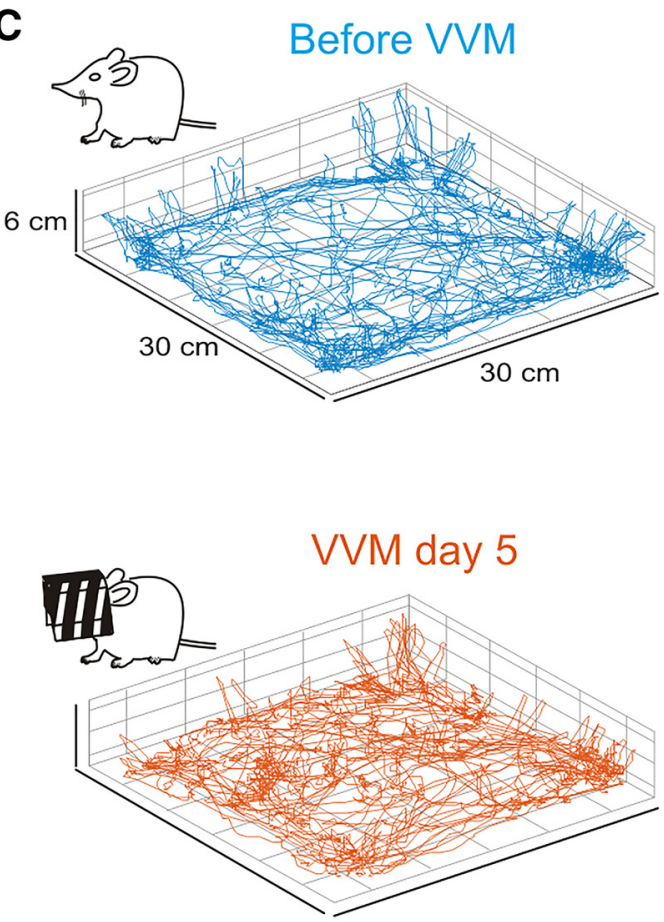
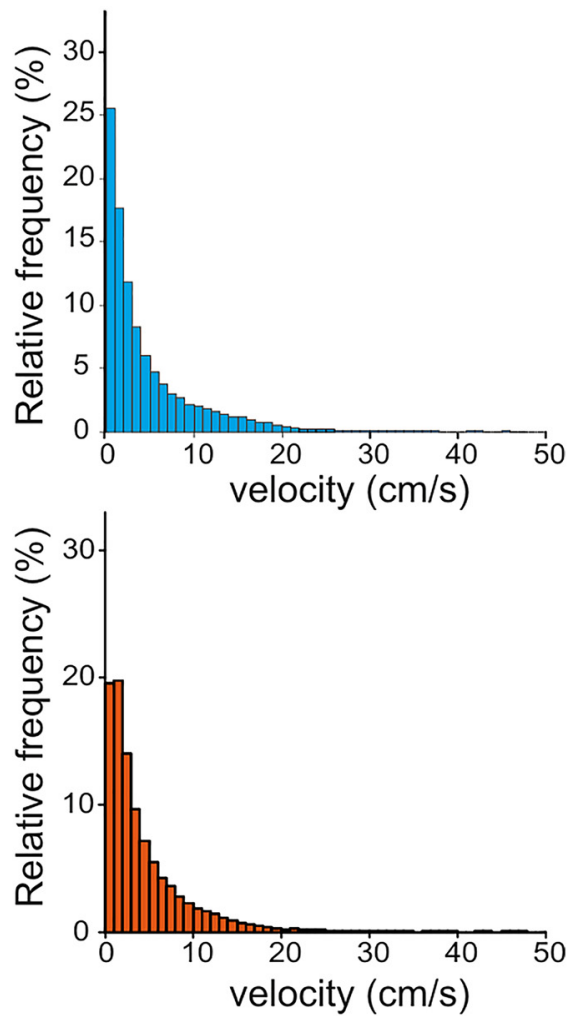

D

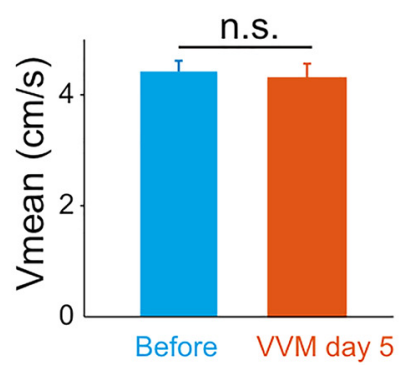

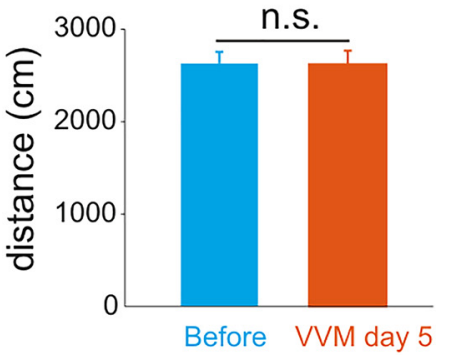

Fig. 2. VVM protocol and open-field experiments. A, Pictures of a mouse during VVM (top) or sham (bottom) protocols. $\boldsymbol{B}$, Mean body weight of sham ( $n=24$, gray line) and VVM mice $(n=57$, red) during the 2 weeks of the protocol. $\boldsymbol{C}$, Locomotion of mice before VVM (top) or after $5 \mathrm{~d}$ of VVM protocol (bottom) recorded while the animal explores the open field. Left panels, examples of $3 \mathrm{D}$ reconstruction of the path of the same animal. Right panels, distribution of velocities for the population of mice $(n=4)$ before VVM (blue) or after $5 \mathrm{~d}$ of VVM (red). $\boldsymbol{D}$. Plots of the mean velocity (Vmean, in $\mathrm{cm} / \mathrm{s}$ ), total covered distance (in $\mathrm{cm}$ ), and vertical explorations (number of rearings) of mice $(n=4)$ before and after $5 \mathrm{~d}$ of VVM. Error bars represent \pm SEM. 
show any sign of disturbed behavior after beginning the protocol. After $2 \mathrm{~d}$, the general behavior of VVM mice returned to normal, with good orientation in the cage, normal locomotion, and social interactions. The increase in weight observed thereafter was comparable to that of sham animals; still, after 2 weeks, a significant weight difference of $\sim 1.5 \mathrm{~g}$ persisted between the two groups (Mann-Whitney, $z=4.28, p=0.00002$ ).

\section{Behavioral measures}

\section{Open-field test}

To determine how the VVM affects the way mice move, 3D tracking during open-field exploratory behavior was performed before the beginning of the protocol and $5 \mathrm{~d}$ after the device was implanted, i.e., once general behavior was back to normal. The setup consisted of a $30-\mathrm{cm}$ cube surrounded by eight CCD cameras (synchronization frequency: $10 \mathrm{~Hz}$; Point Grey Research, GRAS-03K2M). Mice were placed, one at a time, at the center of the arena and left completely undisturbed for $10 \mathrm{~min}$. The 3D trajectory of the center of volume of the mouse was recovered using a multiple-view optical system. The images were electronically synchronized at the frame level using a trigger signal and a time stamp. For each camera, a calibration procedure provided the geometric projection from a 3D reference frame to the 2D image plane using a pinhole model. The 3D reference frame was oriented so that the $X Y$-plane corresponds to the ground plane $(Z=0)$ and the $Z$-axis to the up vertical axis. For each image, a background subtraction technique isolated the 2D silhouette of the mouse. The centroid of the silhouette provided a 2D cue of the animal's center of mass. After calibration of the cameras, the 2D centroids of each frame were triangulated using a direct linear transformation technique to compute the 3D trajectory of the center of volume. The third vertical coordinate was used to count the number of rearings. This experiment allowed analysis of the wholebody velocity, total distance traveled, and number of rearings performed during exploration of the open field.

\section{Video-oculography and vestibulo-ocular performance}

Video-oculography was performed to quantify the gaze-stabilizing reflexes. Mice were head-fixed at a $\sim 30^{\circ}$ nose-down position to align the horizontal canals in the yaw plane (Calabrese and Hullar, 2006; Oommen and Stahl, 2008) and placed in a custom-built Plexiglas tube secured on the superstructure of a vestibular stimulator. VOR performance was tested before and after the VVM protocol, with all sources of light turned off except for the computer screen. The turntable was further surrounded with a closed black box to isolate the animal from any remaining light, with an intensity inside the box $<0.02$ lux. Ten minutes before the experiment, $2 \%$ pilocarpine was applied to the eye to keep the pupil size constant (van Alphen et al., 2010). To record the effect of the VVM on the VOR, the head-fixed animal was put on the vestibular turntable immediately after the removal of the device while kept in the dark to avoid visual access in the environment.

Eye movements were recorded using an infrared video system (ETL-200, Iscan). The video-oculography calibra- tion procedure was similar to that described by Stahl (2004). Eye and head position signals were sampled at 1 $\mathrm{kHz}$, digitally recorded (CED power1401 MkII) with Spike 2 software, and later exported to Matlab for offline analysis (Matlab, The MathWorks; RRID: SCR:001622). Horizontal eye and head movement data were digitally low pass-filtered (cutoff frequency: $40 \mathrm{~Hz}$ ), and position data were differentiated to obtain velocity traces. Segments of data with saccades were excluded from VOR slow-phase analysis. For horizontal sinusoidal rotations, at least 20 cycles were analyzed for each frequency. VOR gain and phase were determined by least-squares optimization:

$$
E H_{v}(t)=g \times\left\{\left[H H_{v} \times\left(t-t_{d}\right)\right]+C^{t e}\right\},
$$

where $E H_{v}(t)$ is eye horizontal velocity, $g$ (gain) is constant value, $H H_{v}$ is head horizontal velocity, $t_{d}$ is the dynamic lag time (in $\mathrm{ms}$ ) of the eye movement with respect to the head movement, and $C^{t e}$ is an offset. $t_{d}$ was used to calculate the corresponding phase $\varphi^{\circ}$ of eye velocity relative to head velocity. The varianceaccounted-for (VAF) of each fit was computed as

$$
1-\left[\frac{\operatorname{var}(e s t-E H v)}{\operatorname{var}(E H v)}\right],
$$

where var represents variance, est represents the modeled eye velocity, and $E H v$ represents the actual eye horizontal velocity. VAF values were typically between 0.70 and 1 ( $\sim 97 \%$ of recordings), where a VAF of 1 indicates a perfect fit to the data. Trials for which the VAF was $<0.5$ were excluded from the analysis.

Overall, the VVM protocol was tested on 25 mice: $n=$ 13 for the characterization of VOR features and $n=12$ for VOR dependency on rotation frequency. Horizontal VOR in the dark was first tested during sinusoidal angular rotation around the vertical axis $(0.5 \mathrm{~Hz}$; velocity in range $20-50 \%$ s) on 13 mice after VVM and on six sham mice. The ratio of slow-phase reduction was calculated by dividing the post-VVM measure by the pre-VVM measure. To compare how VVM affects slow phases versus quick phases, quick-phase analysis was performed at the highest tested velocity $(0.5 \mathrm{~Hz} ; 50 \%$ s). A total of $20-25$ cycles were analyzed in 10 of $13 \mathrm{VVM}$ animals that had a VOR reduction $>60 \%$. The number of quick phases was counted, and their amplitudes were analyzed in Matlab.

To further characterize the slow-phase reduction, the dependence on the stimulation frequency on 12 additional VVM and six sham mice were tested at different frequencies of $0.2,0.5,1$, and $2 \mathrm{~Hz}$ and at a fixed peak velocity of $30 \%$ s.

\section{Optokinetic reflex performance and flocculi shutdown experiments}

To test whether the VOR reduction depends on the cerebellum or on a different brain structure, a flocculus shutdown experiment was performed. During these tests, the OKR was recorded to validate the effect of the pharmacological inhibition of the flocculus/paraflocculus complex. To record the OKR, the mouse was surrounded by a $40-\mathrm{cm}$-wide dome, and sources of light were turned off 
except for the optokinetic projector. The light intensity inside the dome during OKR testing was measured at 185 lux (Luxmeter Lux-1337 Iso-tech). The optokinetic fullfield stimulation was performed by projecting a dot pattern at velocities of $7.5 \%$ in both clockwise and counterclockwise directions. The dot pattern consisted of 25,000 white dots ( $\max$ width $0.075^{\circ}$ ) randomly distributed on a black background. Optokinetic constant velocity stimulations lasted $1 \mathrm{~min}$ and were separated by at least 2 min of darkness. Optokinetic responses were analyzed offline after being imported into Matlab. Segments of data with saccades were excluded from the analysis. Optokinetic responses were measured as the mean eye velocities on segments longer than $1 \mathrm{~s}$. Optokinetic gains were then calculated as the ratios of the mean eye velocities to the constant drum velocity.

After 2 weeks of VVM, both OKR and VOR performances were tested on seven mice. OKR and VOR were again measured $30 \mathrm{~min}$ after the stereotaxic injection of lidocaine ( $n=5 ; 1 \mu \mathrm{L}, 2 \%$ lidocaine) or sham injections of vehicle $(n=2)$ in the bilateral flocculi following a medial (3 $\mathrm{mm}$ medio-lateral) dorso-ventral direct approach $(6 \mathrm{~mm}$ antero-posterior; The Mouse Brain in Stereotaxic Coordinates, Paxinos and Franklin) as in Shutoh et al. (2006). Injection was performed under isoflurane anesthesia to allow a rapid recovery and eye movement measurements. VOR testing was performed after OKR measures in complete darkness at frequencies of $0.2,0.5,1$, and $2 \mathrm{~Hz}$ and at a fixed peak velocity of $30 \%$, as described above.

To confirm the injection was made in the flocculi, fluorescent dye (1\% fluorescein isothiocyanate, Invitrogen) was added to the lidocaine or saline injection and visualized using an epifluorescent microscope on subsequently cut slices, following the procedure described by Okamoto et al. (2011). After intracerebellar injections, intracardiac perfusions were performed, and the brains were removed. The brains were postfixed overnight in PFA 4\% and dehydrated in $30 \%$ sucrose solution for at least 48 h. Then, $80-\mu \mathrm{m}$ brain slices were cut using a microtome and visualized with the epifluorescent microscope (Olympus BX-61).

\section{Electrophysiological experiments}

\section{Whole-cell patch-clamp recordings}

Brain dissections and patch-clamp recordings were performed on slices taken from control $(n=36)$ or VVM $(n=38)$ animals. After decapitation under deep anesthesia (pentobarbital $100 \mathrm{mg} / \mathrm{kg}$ ), the brain was quickly removed and placed in ice-cold, phosphate/bicarbonatebuffered artificial cerebrospinal fluid (ACSF), which included (in mM) 240 sucrose, $2.5 \mathrm{KCl}, 1 \mathrm{NaH}_{2} \mathrm{PO}_{4}, 25$ $\mathrm{NaHCO}_{3}, 3 \mathrm{MgCl}_{2}$, and 10 glucose and was supplemented with $95 \% \mathrm{O}_{2}-5 \% \mathrm{CO}_{2}$. Brainstem slices of 220 $\mu \mathrm{m}$ containing the medial vestibular nuclei (MVN) were cut using a microslicer (Leica). To optimize the maintenance of vestibular afferent fibers in the plane of the slice, an angle of $15^{\circ}$ was added to the standard coronal plane. Slices were then transferred into an incubating vial filled with regular ACSF containing (in mM) $120 \mathrm{NaCl}, 2.5 \mathrm{KCl}, 1$ $\mathrm{NaH}_{2} \mathrm{PO}_{4}, 25 \mathrm{NaHCO}_{3}, 2.5 \mathrm{CaCl}_{2}, 2 \mathrm{MgCl}_{2}$, and 10 glu- cose and oxygenated with $95 \% \mathrm{O}_{2}-5 \% \mathrm{CO}_{2}(\mathrm{pH} 7.4)$. The more rostral slices containing the $\mathrm{MVN}$, in which the brainstem was attached to the cerebellum $(5.8-6.5 \mathrm{~mm}$ AP; The Mouse Brain in Stereotaxic Coordinates, Paxinos and Franklin), were selected and placed in the recording chamber maintained at $32-34^{\circ} \mathrm{C}$. Slices were superfused with regular ACSF at a constant flow rate of $3 \mathrm{ml} / \mathrm{min}$. Patch-clamp pipettes were pulled from borosilicate glass tubing to a resistance of 4-7 M $\Omega$. The internal solution (Sekirnjak and du Lac, 2006) contained (in mM) 140 K-gluconate, $2 \mathrm{MgCl}_{2}, 5 \mathrm{KCl}, 10 \mathrm{HEPES}, 0.03 \mathrm{CaCl}_{2}, 0.1$ EGTA, $4 \mathrm{Na}_{2} \mathrm{ATP}$, and $0.4 \mathrm{Na}_{2} \mathrm{GTP}$ (adjusted to $\mathrm{pH} 7.3$ with $\mathrm{KOH}$ ).

MVN neurons were visualized with a microscope (Olympus BX-51) using differential interference contrast illumination with Nomarski optics. Using the boundaries of the fourth ventricle as landmarks, MVN neuron recordings were restricted to the dorsal half of the nucleus (depth of $4-4.25 \mathrm{~mm}$ ) and to the medial two thirds of the nucleus (0.25-0.8 mm lateral; The Mouse Brain in Stereotaxic Coordinates, Paxinos and Franklin). Neurons at the edge of the fourth ventricle were not recorded. After the surface of the soma of a neuron was approached with a pipette, suction was applied until a giga-ohm seal was made. Recordings were made with a Multiclamp 700B (Molecular Devices). Inhibitory transmission through glycine and $\mathrm{GABA}_{\mathrm{A}}$ receptors was blocked using $10 \mu \mathrm{M}$ strychnine and $100 \mu \mathrm{M}$ picrotoxin, respectively (Sigma-Aldrich; McElvain et al., 2010). The spontaneous discharge was first recorded in current-clamp mode for few minutes until a stable level was reached. MVN neurons that had a membrane potential less than $-45 \mathrm{mV}$ and a spike amplitude $>45 \mathrm{mV}$ were selected for current and voltage clamp experiments. The current and voltage from the amplifier were low-pass filtered at $2 \mathrm{kHz}$ and digitized at $5 \mathrm{kHz}$ (BNC-2090 + PCl-6052E, National Instruments). Custom-written codes in Matlab were used for acquisition and offline analysis.

\section{Excitatory postsynaptic current and plasticity recordings}

Excitatory postsynaptic current (EPSC) recordings were performed on brain slices cut from 12 control mice $(n=17$ neurons; 13 from 8 naive mice and 4 neurons from 4 sham mice) and 19 VVM animals ( $n=31$ neurons). After recording the spontaneous discharge and action potentials at the basic membrane potential, the neuron was clamped at $-70 \mathrm{mV}$. First, the vestibular afferents were stimulated with a concentric bipolar stimulating electrode (FHC) placed on the vestibular fiber bundle. Electrode placement was always made at the same optimal location 4.5 $\mathrm{mm}$ ventral to the horizontal plane and $\sim 1.7 \mathrm{~mm}$ lateral (The Mouse Brain in Stereotaxic Coordinates, Paxinos and Franklin), similar to the procedure followed by McElvain et al. (2010). EPSC were evoked (eEPSC) using stimulation at a frequency of $0.067 \mathrm{~Hz}$ (one stimulation every 15 s). First, the stimulation amplitude was set at $300-400 \mathrm{pA}$ to maximize the EPSC amplitude. Then, after $3 \mathrm{~min}$ of recording, a long-term depression (LTD) protocol consisting of 30 repetitions of 550-ms vestibular nerve stimulation at $100 \mathrm{~Hz}$ was applied according to the procedure provided by McElvain et al. (2010). 
Table 1. Statistical table.

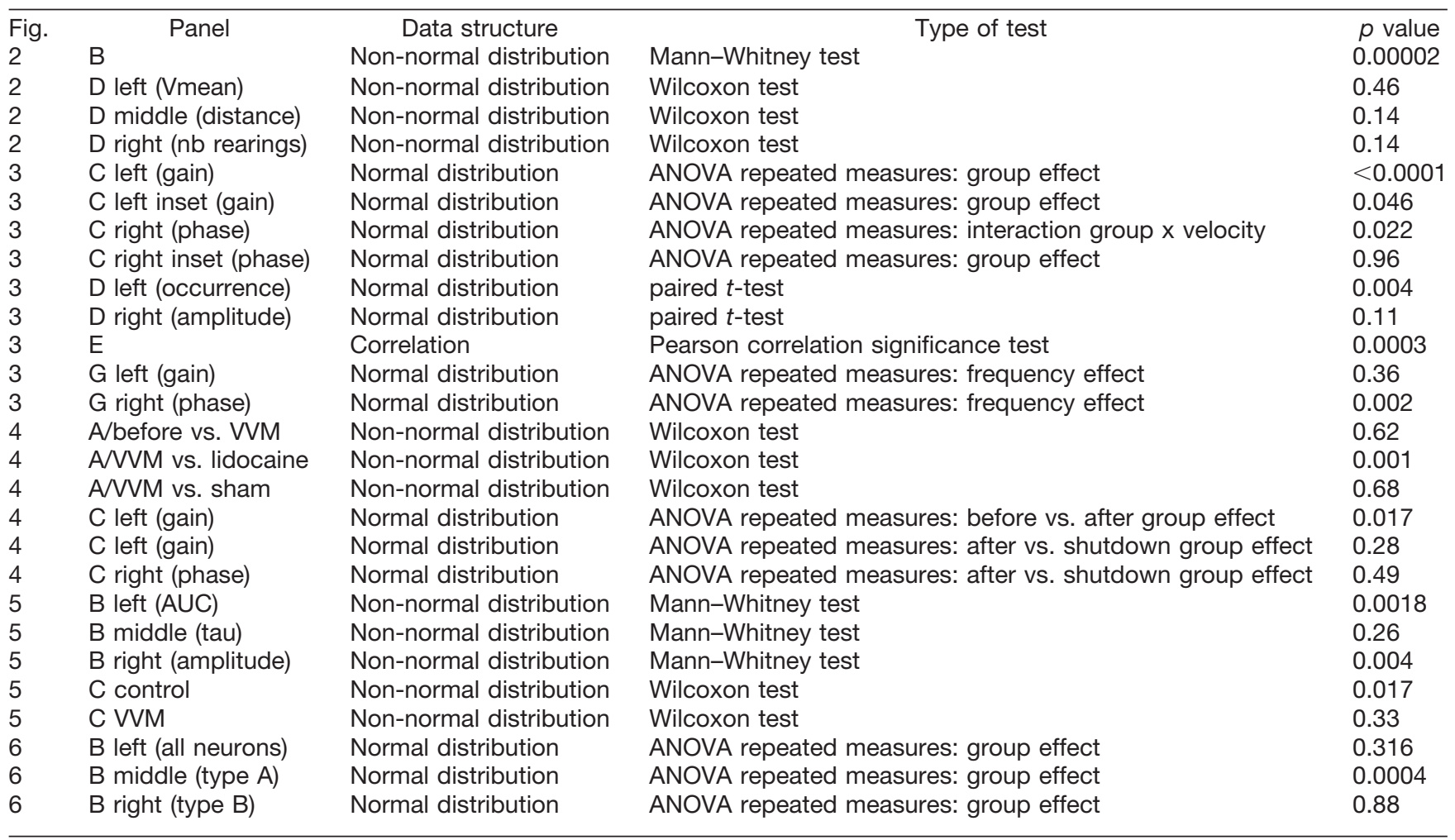

All analyses were performed offline in Matlab. eEPSC amplitude $(\mathrm{pA})$, area under the curve (AUC, $\mathrm{fC}$ ), and time constant $(\tau, \mathrm{ms})$ were calculated offline. For the LTD proto$\mathrm{col}$, eEPSCs were normalized to the preprotocol baseline, and the mean value collected during the $15 \mathrm{~min}$ after the LTD protocol was calculated for graphic representation.

\section{Electrophysiological properties of MVN neurons}

Basic and firing properties of MVN neurons were investigated in current-clamp $(n=63$ control neurons from 36 mice and $n=60 \mathrm{VVM}$ neurons from 38 mice). Because most MVN neurons are spontaneously active in slices, the potential was low-pass filtered at $1 \mathrm{~Hz}$ to obtain an estimate of its average resting level that was taken as the "mean average membrane potential" ( $\mathrm{Vm}$, in $\mathrm{mV}$ ) of each neuron. This membrane potential value was corrected offline by measuring and subtracting the extracellular voltage offset found after withdrawal of the electrode from each neuron. Averages of the spike shapes and following interspike interval profiles were analyzed to obtain the spontaneous firing rate (in spikes/s), the associated coefficient of variation (CV), the amplitude of the afterhyperpolarization (AHP, in $\mathrm{mV}$ ), the spike threshold potential (in $\mathrm{mV}$ ), the concavity, and the convexity (in $\mathrm{mV}$ ). The AHP and interspike interval first derivative was used to quantify the amplitude of the double AHP (dAHP, in V/s) and the presence of an A-like rectification (AHPR in V/s). The classification of MVN neurons was performed based on these quantitative criteria following the procedure provided by Beraneck et al. (2003). Excitability of the neurons was tested using injection of hyperpolarizing/depolarizing steps from basic potential with steps of currents $(1 \mathrm{~s}$;
$25 \mathrm{pA}$ increment, range $\pm 125 \mathrm{pA}$ ). Excitability was calculated as the mean firing frequency during the steps (in spikes/s). Membrane resistance was calculated from the hyperpolarizing step at $-75 \mathrm{pA}$.

\section{Statistical analyses}

Statistical analyses were performed using Statistica 7.1 software (StatSoft). A statistical table is provided (see Table 1). Repeated-measures ANOVAs were performed on VOR gain and phase across frequencies or velocities. Nonparametric unpaired Mann-Whitney tests were performed to compare measures between control and WM mice. Nonparametric paired Wilcoxon signed rank tests were performed to compare EPSC amplitude before and after the LTD protocol.

\section{Results}

A new protocol was developed to expose freely behaving mice to a VVM for a 2-week period. The consequences of the VVM on locomotor behavior and its effects on the efficacy of the VOR were first tested. Next, the neural changes underlying long-term VOR reduction were studied using whole-cell patch-clamp electrophysiology on brainstem slices.

\section{Behavioral experiments \\ Open-field data}

During the freely behaving VVM protocol, the animal generates active movements. To determine whether the implanted device modifies the way the animal moves, the exploratory behavior of four mice was analyzed using a 3D tracking video system. When placed in the open field, mice naturally explored the environment in both horizontal 
and vertical planes (Fig. 2C, left). Exploratory behavior was compared for each mouse before the beginning the protocol (blue trace) and $5 \mathrm{~d}$ after the device was implanted (red trace), while the mouse was still wearing the device. Overall, we observed no difference in the way the mice explored the open field. The distribution and range of walking velocities were comparable (Fig. $2 C$, right), and there was no difference in the mean velocity $(n=4$, Wilcoxon test, $z=0.73, p=0.46$ ), total covered distance ( $n=4$, Wilcoxon test, $z=1.46, p=0.14$ ), or number of vertical explorations (rearings; $n=4$, Wilcoxon test, $z=$ 1.46, $p=0.14$; Fig. 2D). These data complement our general observations (see Materials and methods; Fig. $2 B$ ), which suggest that after the initial $48 \mathrm{~h}$, mice wearing the VVM device display relatively normal behavior.

\section{VWM protocol reduces the VOR}

To evaluate the effects of the VVM on gaze stabilization, the VOR was quantified using video-oculography in the dark during sinusoidal rotations around a vertical axis (Fig. $3 A$ ). The VOR of 25 mice was compared before the implantation of the VVM device and immediately after its removal 2 weeks later; it was similarly measured on six sham mice. As a result of VVM, the amplitude of the eye movements observed during table rotations was greatly reduced (Fig. 3B, response of the same mouse before and after WVM). A first group of 13 mice was tested at a fixed frequency of $0.5 \mathrm{~Hz}$ and peak velocities from 20 to $50 \%$ s. A decrease of the VOR gain by $>50 \%$ was observed for all conditions after the 2 weeks of VVM (Fig. 3C, left, repeated-measures ANOVA, group effect, $F_{1,24}=42.6, p<0.0001$ ), with a shift toward phase lead at velocities $<40 \%$ s (Fig. $3 C$, right, repeatedmeasures ANOVA, group $\times$ velocity interaction effect, $F_{3,72}$ $=3.41, p=0.022)$. In contrast, sham mice $(n=6)$ exposed to a comparable experience in the absence of VVM had no reduction in VOR (slight increase in VOR gain: repeatedmeasures ANOVA, group effect, $F_{1,5}=6.92, p=0.046$; VOR phase: repeated-measures ANOVA, group effect, $F_{1,5}=$ $0 ; 003, p=0.96$; see traces in Fig. $3 B, C$ ). In addition to the slow-phase changes, quick phases were also investigated. After VVM, the occurrence (Fig. 3D, left) of the quick phases was significantly diminished $(n=10$, paired $t$-test, $t=3.77$, $p=0.004$ ) and although there was a tendency of decreased amplitude of saccades after WVM, this difference did not reach statistical significance (Fig. 3D, right; $n=10$, paired $t$-test, $t=1.77, p=0.11$ ). However, the comparison of the changes in slow phases and quick phases demonstrated a significant correlation (Fig. 3E; $R=0.905 ; z=1.50, p=$ 0.0003 ), suggesting that both features of the vestibuloocular reflex were modified by the WVM.

Because the WVM was generated by an actively behaving mouse, we then questioned how this reduction affects the VOR across different frequencies. The dependence of the VOR reduction on the stimulating frequency was therefore tested on the six sham animals and 12 additional VVM mice at four different frequencies $(0.2,0.5,1$, and $2 \mathrm{~Hz}$; fixed peak velocity of $30 \% / \mathrm{s})$. No differences were found in the VOR gain and phase of sham animals before and after the protocol (mean gain \pm SD before/after: $0.2 \mathrm{~Hz}, 0.14 \pm 0.05 / 0.16 \pm$ $0.06 ; 0.5 \mathrm{~Hz}, 0.34 \pm 0.05 / 0.32 \pm 0.08 ; 1 \mathrm{~Hz}, 0.50 \pm 0.09 /$ $0.55 \pm 0.1 ; 2 \mathrm{~Hz}, 0.59 \pm 0.18 / 0.55 \pm 0.13$; repeated-
A

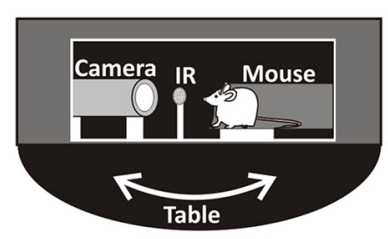

C
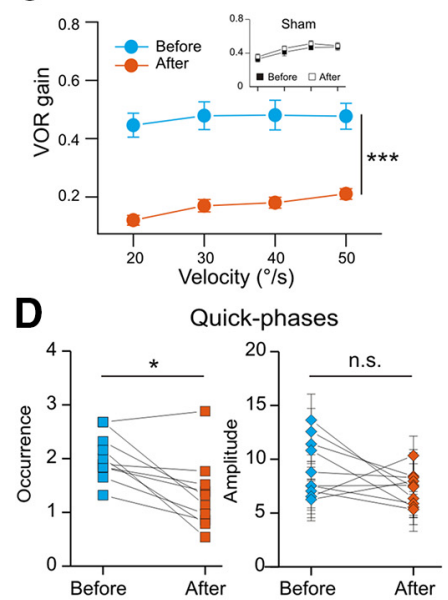

$\mathbf{F}$
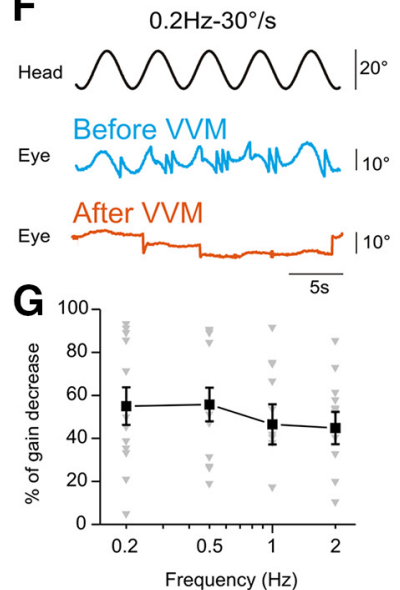

$\mathbf{E}$
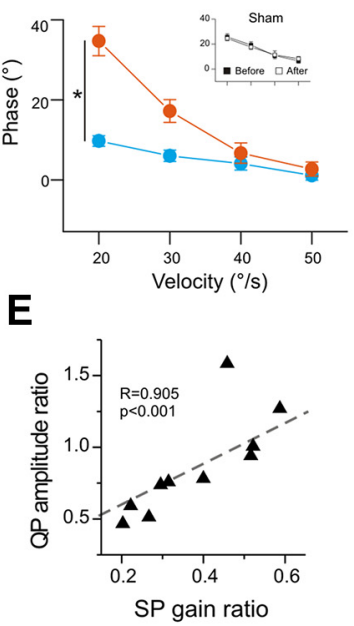

Head Mm

Before VVM

Eye mi2

After VVM

Eye $12^{\circ}$

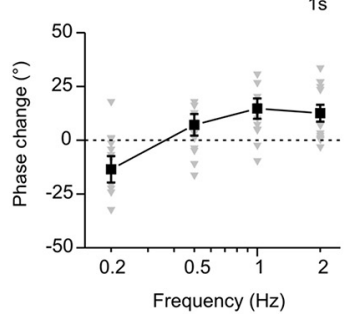

Fig. 3. VOR reduction. $\boldsymbol{A}$, Illustration of the setup used to test the VOR. IR, infrared light. $\boldsymbol{B}$, Example raw traces of the VOR in the dark recorded before (blue line) and after 2 weeks of VVM (red line) from the same animal. Gray trace, sham mouse tested after 2 weeks of wearing the helmet. White arrows indicate example of quick phases observed after VVM. Head and eye traces show rightward movements in the upward and downward directions, respectively. $\boldsymbol{C}$, Mean VOR gain (left) and corresponding phase (right) plotted as a function of tested velocity ( $n=13$ mice; fixed frequency of $0.5 \mathrm{~Hz}$ ) measured before (blue lines) and after 2 weeks of VVM (red lines). Insets: sham ( $n=6$ mice) before (filled squares) and after (empty squares) the protocol. $\boldsymbol{D}$, Occurrence (left) and amplitude (right) of quick phases ( $n=10$ mice). $\boldsymbol{E}$, Quick-phase amplitude ratio (after/before values) is significantly correlated to slow-phase gain ratio $(* * * p<0.001)$. $\boldsymbol{F}$, Example raw traces of the VOR reduction at 0.2 or $2 \mathrm{~Hz}$ stimulation. $\mathbf{G}$, Mean percentage of gain decrease (left, $n=12$ ) or phase change (right, $n=12$ ) depending on stimulating frequencies. The gray triangles represent the individual values, and the black lines represent the mean values. Error bars represent \pm SEM. 
A

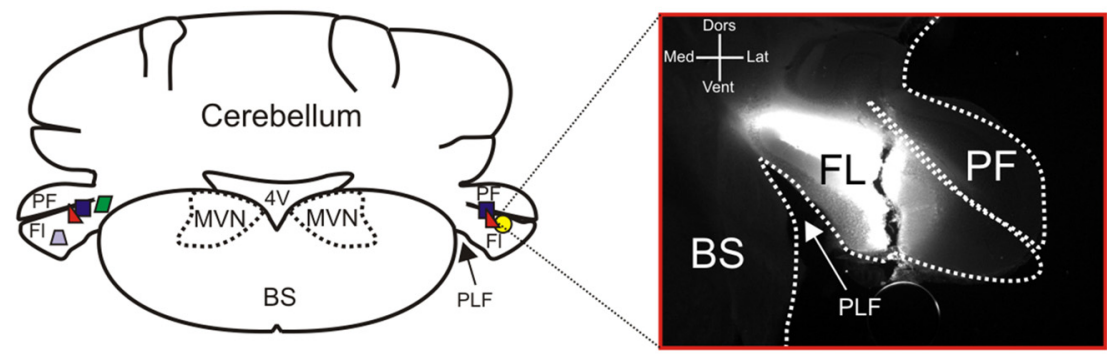

B
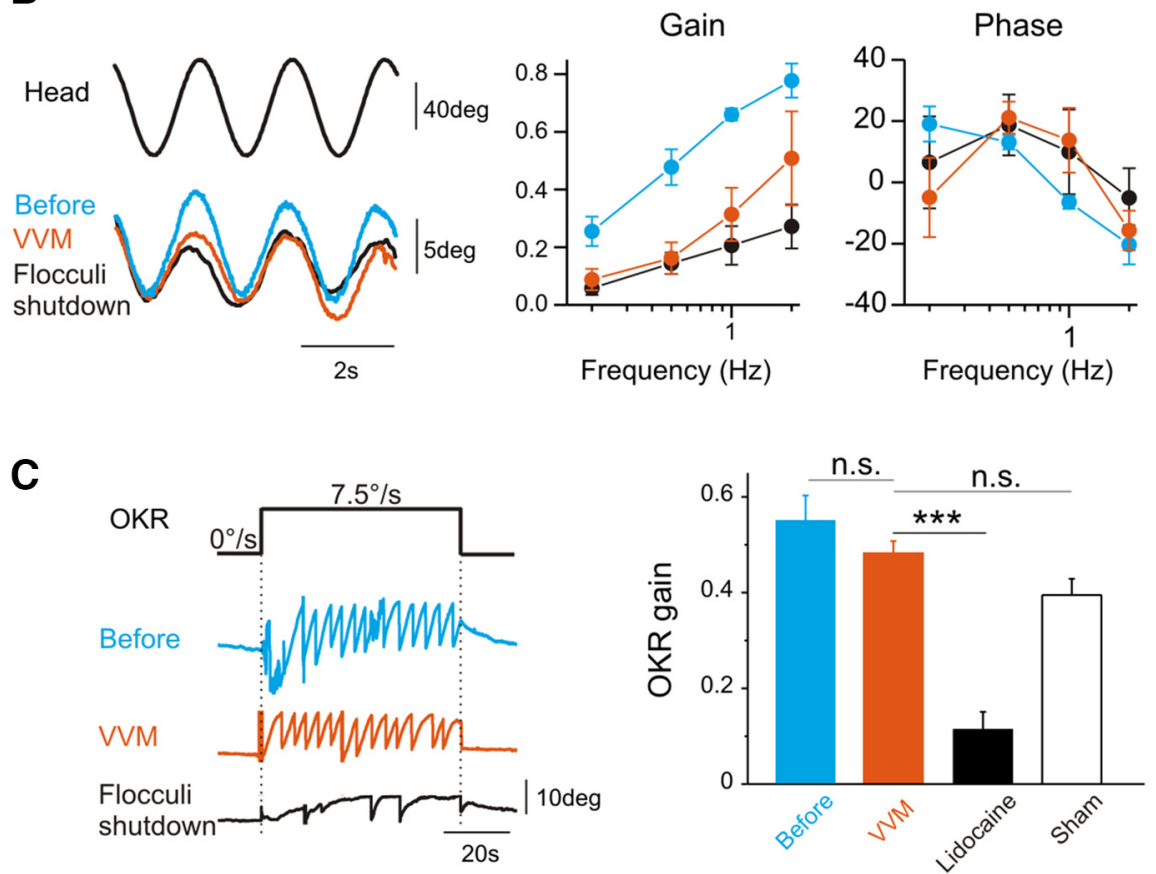

Fig. 4. Flocculi shutdown experiments. $\boldsymbol{A}$, Left, coronal section of the brainstem and cerebellum illustrating the lidocaine injections in flocculi complex. PF, paraflocculus; FI, flocculus; PLF, posterolateral fissure; 4V, 4th ventricle; BS, brainstem. Right, example of a lidocaine injection coupled to fluorescein isothiocyanate. Dors, dorsal; Vent, ventral; Med, medial; Lat, lateral. $\boldsymbol{B}$, Left, example raw traces of VOR in the dark recorded before (blue line), after 2 weeks of VVM (red line), and after flocculi shutdown (black line). All traces are from the same animal. Right, Bode plots of VOR gain and phase ( $n=5$ mice). $\boldsymbol{C}$, Left, example raw traces of eye movements recorded during optokinetic stimulation (60s-long full-field stimulation at $7.5 \%$ s constant velocity). All traces are from the same animal. Right, mean OKR gain recorded before, after VVM, after lidocaine injection, or on sham animals. Error bars represent \pm SEM.

measures ANOVA, group effect; for VOR gain: $F_{1,5}=0.007$, $p=0.94$; for VOR phase: $F_{1,5}=0.99, p=0.36$ ). Fig. $3 F$ shows examples traces of the VOR generated at frequencies of $0.2 \mathrm{~Hz}$ (left) or $2 \mathrm{~Hz}$ (right) before and after VVM. Again, we found a significant decrease of the gain of the VOR at all frequencies after VVM, represented by a percentage of gain decrease of $\sim 50 \%$. Although the average decrease of the VOR was comparable at all frequencies (repeated-measures ANOVA, frequency effect, $F_{3,33}=1.11, p=0.36$ ), Fig. $3 G$ illustrates the variability in the amount of VOR decrease between individuals (gray triangles). The long-term VVM also had a significant effect on the phase of the VOR, with a shift toward greater phase lag at $0.2 \mathrm{~Hz}$ and toward greater phase lead at frequencies $\geq 0.5 \mathrm{~Hz}$ (Fig. 3G, right, repeatedmeasures ANOVA, frequency effect, $F_{3,33}=6.30, p=$ $0.002)$. Overall, video-oculography results clearly demon- strate that the 2 weeks of WM protocol led to a strong gain-down reduction of the VOR.

\section{Flocculus shutdown experiment}

To determine whether the long-term VOR reduction depends on cellular changes located at the level of the cerebellum or in downstream structures, flocculus shutdown experiments were performed by injection of lidocaine (Fig. 4). Injections of lidocaine were coupled to fluorescent dye to validate a posteriori the location in the flocculi (Fig. 4A). As described above, VOR gain significantly decreased after 2 weeks of VVM (Fig. $4 B, n=5$; repeated-measures ANOVA, before vs. after group effect, $F_{1,6}=10.52, p=$ $0.017)$. When the flocculi were inactivated, the gain and phase of the VOR remained unchanged compared to the values reached after VVM (Fig. 4B; repeated-measures 
Table 2. Static intrinsic properties of MVN neurons.

\begin{tabular}{|c|c|c|c|c|c|c|c|c|c|c|}
\hline \multirow{3}{*}{ Property } & \multicolumn{2}{|c|}{ All } & \multicolumn{2}{|c|}{ type $A$} & \multicolumn{2}{|c|}{ type B } & \multirow{2}{*}{\multicolumn{4}{|c|}{$\begin{array}{c}\text { Statistical tests (Mann-Whitney) } \\
\text { Control vs. VVM }\end{array}$}} \\
\hline & Control & VVM & Control & VVM & Control & VVM & & & & \\
\hline & 63 & 60 & 27 & 17 & 36 & 43 & All & & type A & type B \\
\hline Membrane potential (mV) & -48.28 & -49.00 & -47.24 & -47.22 & -49.07 & -49.70 & $z=-1.09$ & NS & $z=-0.02 \mathrm{NS}$ & $z=-0.81 \mathrm{~N}$ \\
\hline Spike threshold (mV) & -31.32 & -32.34 & -29.58 & -30.73 & -32.63 & -32.97 & $z=-1.36$ & NS & $z=-0.99 \mathrm{NS}$ & $z=-0.39 \Lambda$ \\
\hline Firing rate $(\mathrm{Hz})$ & 12.24 & 7.85 & 13.83 & 8.14 & 11.04 & 7.74 & $z=-2.49$ & $p=0.013$ & $z=-1.73 p=0.08$ & $z=-1.57 \wedge$ \\
\hline Coefficient of variation & 0.16 & 0.22 & 0.15 & 0.22 & 0.17 & 0.22 & $z=1.97$ & $p=0.048$ & $z=1.40 \quad N S$ & $z=1.11 \quad N$ \\
\hline $\operatorname{AHPR}(\mathrm{V} / \mathrm{s})$ & 0.20 & 0.21 & 0.44 & 0.67 & 0.02 & 0.03 & $z=-1.29$ & NS & $z=-0.09 \mathrm{NS}$ & $z=-0.11 \mathrm{~N}$ \\
\hline dAHP (V/s) & 0.71 & 0.69 & 0.00 & 0.00 & 1.24 & 0.96 & $z=-0.07$ & NS & NA & $z=-1.14 \Lambda$ \\
\hline $\mathrm{AHP}(\mathrm{mV})$ & 26.11 & 25.25 & 29.68 & 28.54 & 23.43 & 23.95 & $z=-0.16$ & NS & $z=-0.14 \mathrm{NS}$ & $z=0.48 \mathrm{~N}$ \\
\hline Concavity (mV) & -1.33 & -1.50 & -2.49 & -3.47 & -0.46 & -0.73 & $z=0.002$ & NS & $z=-1.49 \mathrm{NS}$ & $z=-0.59 \mathrm{~N}$ \\
\hline Convexity (mV) & 0.82 & 0.84 & 0.56 & 0.34 & 1.02 & 1.05 & $z=0.13$ & NS & $z=-0.98 \mathrm{NS}$ & $z=0.03 \quad N$ \\
\hline
\end{tabular}

Parameters of the spontaneous activity of MVN neurons recorded on control and WV slices. Static parameters are based on the analysis of the pacemaker discharge of the neurons and the quantification of the AHP and interspike interval. Neurons are pooled (all) or segmented in type A and type B. Overall, the intrinsic membrane properties recorded in controls and VVM mice show limited differences between type A and type B neurons. AHPR, dAHP, and AHP are parameters representing kinetics of the AHP and interspike interval (see Materials and methods). Statistically significant differences are highlighted in red. NS, not significant.

ANOVA, after vs. flocculi shutdown group effect, on gain measures: $F_{1,8}=1.35, p=0.28$; on phase measures: $F_{1,8}$ $=0.52, p=0.49)$. To functionally confirm the efficacy of the injection, the OKR was measured on the seven mice before and after VVM, and immediately after injection of lidocaine $(n=5)$ or saline $(n=2)$. Before VVM, optokinetic full-field stimulation performed at $7.5 \%$ s triggered a consistent response (Fig. 4C, blue trace). After 2 weeks of VM, optokinetic responses were mostly preserved (Fig. $4 C$, red trace; Wilcoxon test, $p=0.625)$. We noted, however, that OKR responses were sometimes qualitatively less robust than before VVM (not shown). After lidocaine injection in the flocculi, the OKR responses were largely abolished (Fig. 4C, black trace), as demonstrated by the strong reduction of the mean gain after the lidocaine injection (Wilcoxon test, $p<0.001$ ). As expected, sham injection did not significantly modify the OKR responses ( $n=2,0.38 \pm 0.13$, Wilcoxon test, $p=0.686$ ). The OKR tests therefore confirmed the ability of lidocaine injection to inhibit the flocculi. Overall, these experiments demonstrated that after 2 weeks of VVM, the reduction of the VOR depends on plasticity located outside the flocculus/paraflocculus complex.

\section{In vitro electrophysiological recordings}

To determine whether the VOR reduction involves plastic changes at the level of the brainstem, in vitro electrophysiology was performed on mice after the 2 weeks of VM and compared with control mice. Previous studies suggested that the reduction of the VOR could depend on plastic changes in the direct horizontal VOR pathway. Therefore, we measured the synaptic and intrinsic properties of central MVN neurons after VOR reduction.

\section{Synaptic plasticity after VVM protocol}

Whole-cell patch-clamp electrophysiology was performed on central neurons recorded in brainstem slices taken from control and VVM mice. We first recorded evoked eEPSCs from second-order neurons in response to afferent stimulation (vestibular afferent fiber bundle stimulation; Fig. $5 A$, left) in control ( $n=17$ neurons) and VVM ( $n=31$ neurons) conditions. As illustrated in Fig. $5 A$, right, eEPSCs were smaller in VVM neurons compared with control neurons, a result confirmed by an AUC (Fig.
$5 B$, top left) that was significantly smaller in the VVM than in control condition (Mann-Whitney test, $z=3.13, p=$ $0.0018)$. To determine whether this decrease in eEPSC AUC depends on changes in postsynaptic receptors, we explored the kinetic characteristics of the eEPSC. The eEPSC time constant $(\tau)$ was not different between the two groups (Fig. 5B, top middle, Mann-Whitney test, $z=$ $-1.12, p=0.26)$. In contrast, the amplitude was smaller after VVM in comparison with control mice (Fig. 5B, top right, Mann-Whitney test, $z=2.84, p=0.004$ ), as shown in the distribution of eEPSC amplitude shifted toward smaller amplitudes (Fig. 5B, bottom). These results suggest that the receptor units involved in the eEPSC responses are qualitatively not different in VVM mice from controls. Altogether, this experiment suggests that the long-term VOR reduction is associated with a reduction of the efficacy of the synapses between vestibular afferents and central vestibular neurons.

Last, we asked whether additional down-tuning of this synapse was possible or whether the synaptic efficacy was already at its minimum. To address this question, we performed a LTD protocol in both control and VVM mice (Fig. 5C). As previously reported (McElvain et al., 2010), LTD could be experimentally induced in control slices, which led to a significant decrease in the eEPSC amplitude after the stimulation protocol ( $n=8$; Wilcoxon test, $z$ $=2.38, p=0.017$ ). On the other hand, when neurons from VVM mice were tested, no further decrease of the synapse efficacy could be triggered ( $n=14$; Wilcoxon test, $z$ $=0.97, p=0.33$ ).

Overall, these data show that the reduction in the VOR observed after VVM is correlated with a reduction in the efficacy of the synapses between the vestibular nerve and central vestibular neurons. Because no additional decrease of the synaptic efficacy could be triggered experimentally, we then explored whether these synaptic changes were supplemented by a change in the intrinsic excitability of central vestibular neurons.

\section{Intrinsic properties of MVN neurons after VVM}

To explore this hypothesis, we first characterized the membrane properties of the neurons recorded in slices of control and VVM mice (Table 2). Because vestibular neurons have a pacemaker activity in brainstem slices (Dutia 

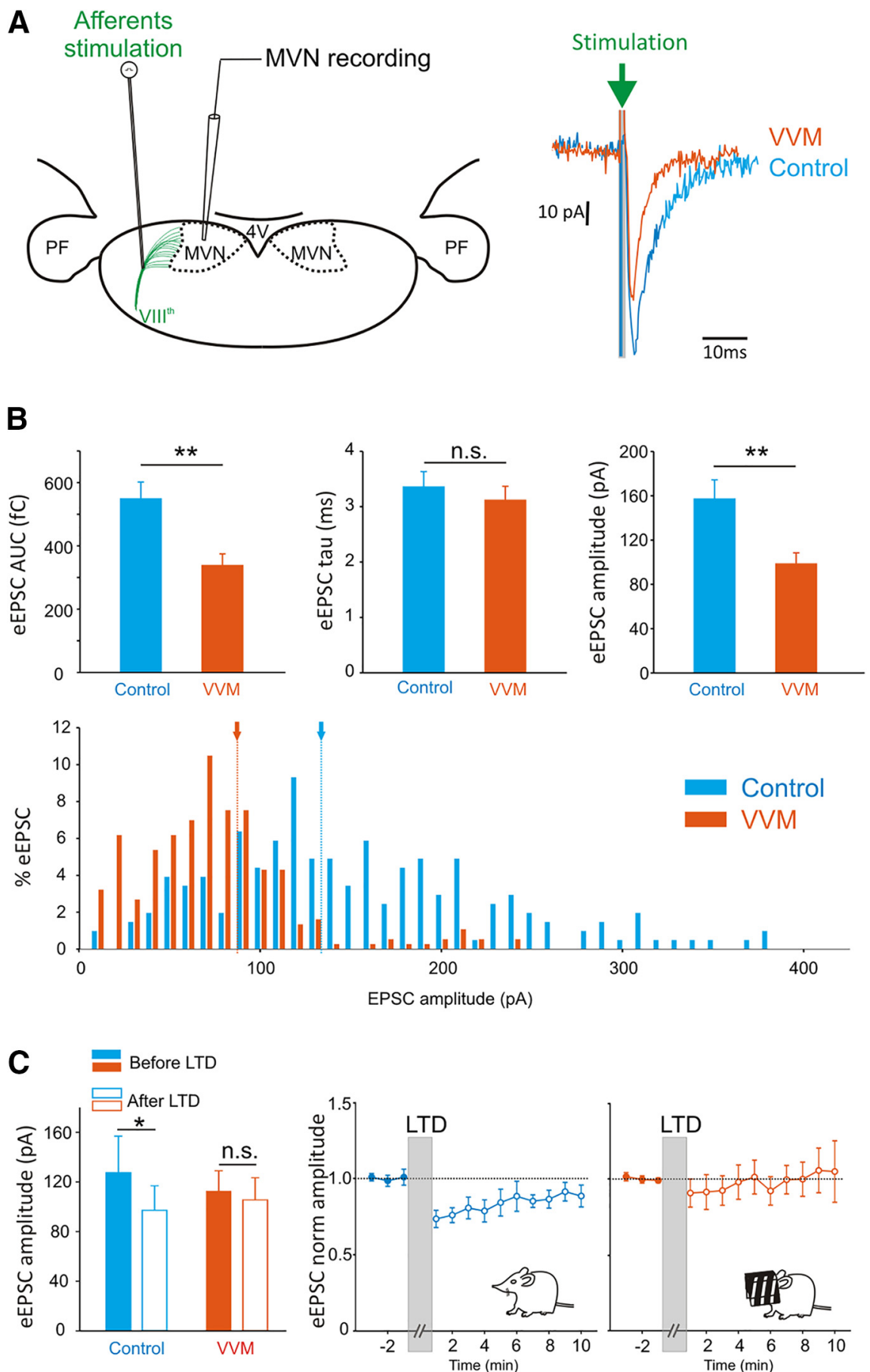

Fig. 5. Stimulation of afferents: vestibular synapses efficacy. $\boldsymbol{A}$, Left, illustration of in vitro patch-clamp recordings of MVN neurons on coronal brainstem slice. The stimulating electrode is placed on the central vestibular fiber bundles. PF, parafloccular region; 4V, 4th ventricle. Right, example raw traces of superimposed eEPSC recorded from a MVN neuron of a control mouse (blue line) and from a mouse after VVM (red line). B, Top, evoked EPSCs AUC (in fC), time constant ( $\tau$, in ms), and amplitude (in pA) recorded from control neurons $(n=17)$ or after VVM $(n=31)$. Bottom, distribution of eEPSC amplitude for control (blue bars) and VVM (red bars) conditions. Arrows and dashed lines indicate the medians ( $\sim 135 \mathrm{pA}$ for control and $\sim 85 \mathrm{pA}$ for VVM). C, Plasticity of vestibular nerve synapses onto MVN neurons. Left, eEPSC amplitude recorded on control $(n=8)$ and VVM $(n=14)$ neurons before (filled bars) and after (empty bars) LTD protocol. Right, mean eEPSC peak amplitude before and after LTD protocol for control (blue line) and VVM (red line) neurons. The eEPSC values are normalized to the mean baseline value before LTD protocol (filled circles). Empty circles represent measures following LTD protocol. Error bars represent \pm SEM. 
A

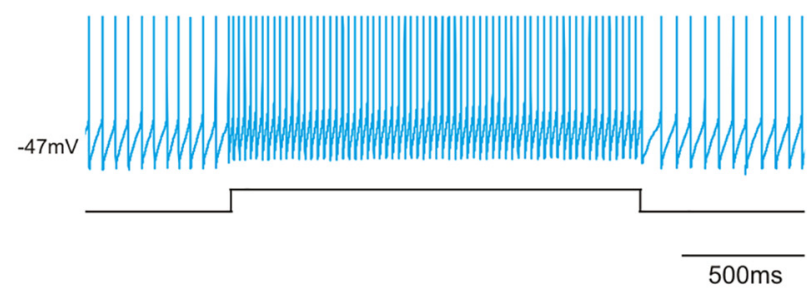

VVM type A

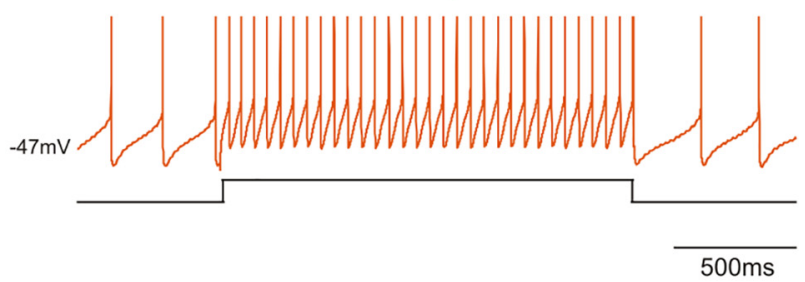

Control type B

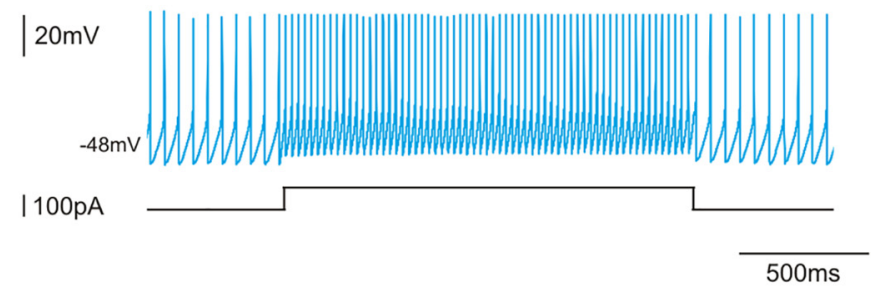

VVM type B

$20 \mathrm{mV}$

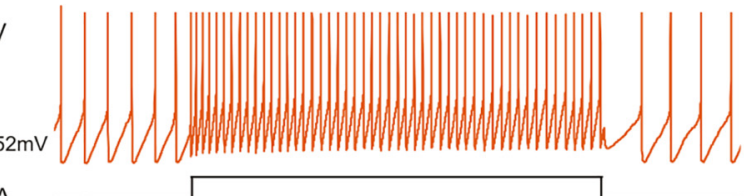

I 100pA
B

\section{All neurons}

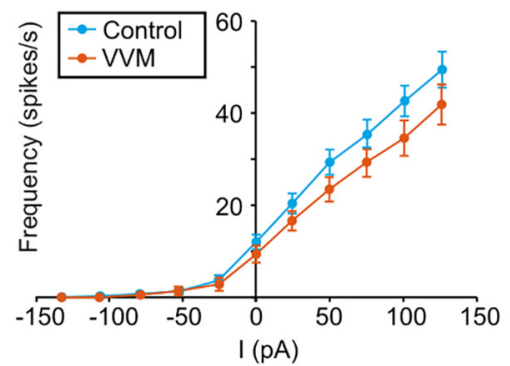

Type A

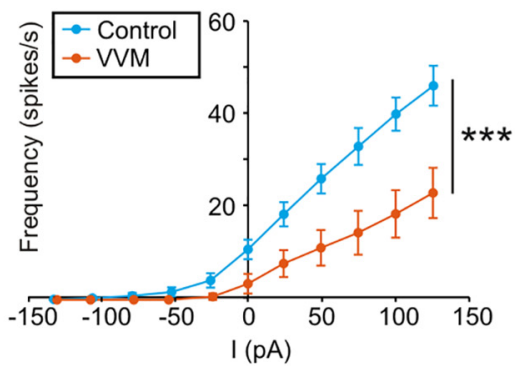

Type B

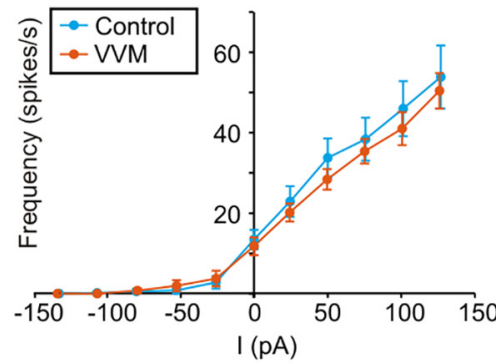

Fig. 6. Excitability of second-order vestibular neurons in response to step-like currents. $\boldsymbol{A}$, Example raw traces of MVN neurons recorded in control (top) type A (left) and type B (right), or VVM (bottom) conditions. B, Relation between the injected current and the frequency of discharge (I/F curves) for all MVN neurons (left, $n=38$ control and $n=24 \mathrm{VVM}$ ), type A (middle, $n=23$ control and $n=7 \mathrm{VVM}$ ) and type B (right, $n=15$ control and $n=17 \mathrm{VVM})$ neurons. ( $* * * p<0.001)$.

et al., 1992, 1995), we first characterized their responses in the absence of external stimulation (Table 2). There was no difference in the spike shape parameters between control and VVM conditions ( $n=63$ control neurons vs. $n=60$ VVM neurons). However, VVM neurons showed a decrease of their spontaneous firing rate in comparison to control neurons (Mann-Whitney test, $z=-2.49, p=$ 0.013 ) and a slightly more regular spontaneous discharge than control neurons (CV of 0.16 vs. 0.22 ; Mann-Whitney test, $z=1.97, p=0.048$ ). MVN neurons were previously shown to be composed of different subpopulations that can be partly segregated using their electrophysiological signature at rest (Straka et al., 2005; Eugène et al., 2011; Beraneck and Idoux, 2012). Hence, we further divided the recorded neurons using the canonical type $A$ and type $B$ classification. This analysis revealed that compared with controls, type A neurons appear to be specifically modified by the VVM, with a tendency for a lower firing rate than control neurons (Mann-Whitney test, $z=-1.73, p=$ 0.08 ).

We then used step-like current stimulation (Fig. 6A) to investigate the excitability of the neurons by assessing their current-frequency relationship (I/F curve). No signif- icant differences in I/F curves between control $(n=38)$ and VVM $(n=24)$ conditions were found when neuronal subtypes were pooled together (Fig. 6B, left; on depolarizing steps, repeated-measures ANOVA, group effect: $\left.F_{1,52}=1.03, p=0.316\right)$. However, when neurons were segmented into type $A$ and type $B$, we found a significant decrease in the excitability restricted to type A neurons ( $n$ $=23$ control, $n=7$ VVM; Fig. 6B, middle; on depolarizing steps, repeated-measures ANOVA, group effect: $F_{1,26}=$ 8.00, $p=0.009$; group $\times$ current interaction: $F_{5,130}=$ 4.88, $p=0.0004$ ), whereas VVM had no significant effect on the excitability of type B neurons ( $n=15$ control, $n=$ 17 VVM; Fig. 6B, right; on depolarizing steps, repeatedmeasures ANOVA, group effect: $F_{1,24}=0.02, p=0.88$ ). We then calculated the resistance of the neurons using hyperpolarizing steps and found that the resistance of both type A neurons and type B was not different in control and VVM neurons (type A control $310 \pm 33$ vs. type A VVM $302 \pm 46 \mathrm{M} \Omega$; type B control $382 \pm 54$ vs. type B VVM $370 \pm 47 \mathrm{M} \Omega$; Mann-Whitney test, $z=-0.11$ for type $A, z=1.10$ for type $B$, not significant).

Overall, these in vitro results demonstrate that after 14 $\mathrm{d}$ of VVM, long-term reduction of the VOR is accompanied 
by changes occurring in the brainstem, within the direct VOR pathway. These changes consist of both a reduction of the efficacy of the vestibular afferents synapses on MVN neurons and changes in the intrinsic membrane properties of some MVN neurons.

\section{Discussion}

Using VVM in freely behaving mice, we showed neural evidence that long-term VOR reduction is correlated to plasticity within the vestibular nuclei.

\section{A new protocol for VOR reduction through visuo- vestibular mismatch}

In humans and monkeys, VOR adaptation is studied by the use of prisms that the subject wears for several days (Berthoz et al., 1981; Melvill Jones et al., 1988; Anzai et al., 2010; Nagao et al., 2013). On the other hand, traditional protocols triggering a VOR gain-down adaptation in rodents involve rotating the head-fixed animal in phase with the visual surround. This basic procedure is then repeated on several consecutive days to drive long-term adaptation (Raymond and Lisberger, 1996; Boyden and Raymond, 2003; Rinaldi et al., 2013). This methodology is timeconsuming and consists of discontinuous training sessions interrupted by intertrial intervals of variable duration (Boyden and Raymond, 2003). It furthermore represents passive learning, since the vestibular stimulation is not actively generated by voluntary movements (Roy and Cullen, 2004; Cullen, 2012). VVM methodology bypasses these experimental constraints. Here, the VOR reduction occurs in response to voluntary natural head movements in an uninterrupted process. Using this approach, we report a general reduction of VOR by $\sim 50 \%$. Our data on open-field locomotion show that mice adapt to the device and that the animals ambulate and explore the environment relatively normally, with no indication of a generalized vestibular impairment. The results obtained on sham animals further demonstrate that the reduction of VOR is a consequence of the visuo-vestibular mismatch and not of a generalized motor impairment. This decrease is comparable to the ones observed using standard VOR gaindown adaptation protocols in head-fixed animals (Boyden et al., 2004; Kassardjian et al., 2005). Moreover, VVM was shown to differentially affect the timing (phase) of the VOR depending on the tested frequency. This result can be interpreted in the framework of frequency-selective channels for vestibular processing (Baker et al., 1981; Lisberger et al., 1983; Straka et al., 2009), and of phase crossover after classic VOR adaptation. Phase crossover after gain-down learning induces phase lags at frequencies below the training frequency and phase leads at frequencies above the training frequency (Lisberger et al., 1983; Raymond and Lisberger, 1996). We show that VVM induces a similar effect on the timing of eye movements. The crossover occurs between 0.2 and $0.5 \mathrm{~Hz}$, which is compatible with the natural range of mouse head movements dominated by low frequencies (Beraneck et al., 2008). Frequencies $<0.5 \mathrm{~Hz}$ also correspond to the range at which the visual information is most important for gaze stabilization (Faulstich et al., 2006). As the changes in eye movements after VVM are comparable to those observed after a standard head-fixed protocol driving gain down, VOR reduction observed in both cases could depend on comparable cellular mechanisms. We note, however, that the VVM constitutes a different protocol from the classic VOR adaptation performed in mice, and that caution should be taken when interpreting results obtained under different experimental conditions.

\section{The role of brainstem changes in long-term VOR reduction}

The hypothesis of a transfer of memory from the cerebellum to downstream structures has been explored using different types of cerebellar-dependent motor learning. Eyelid conditioning protocols have demonstrated that the initiation of learning in the cerebellar cortex (Ohyama and Mauk, 2001) is followed by the induction of plasticity in downstream nuclei for consolidation (Kleim et al., 2002; Ohyama et al., 2006). A comparable sequence of memory formation was demonstrated using optokinetic gain-up learning (Shutoh et al., 2006; Okamoto et al., 2011). OKR studies also suggested that long-term motor learning could depend on plastic processes within the vestibular nuclei (Shutoh et al. 2006). Adaptation of the VOR has been proposed early on to depend on several sites of plasticity in the cerebellum and the brainstem (Lisberger et al., 1981, 1994; Broussard and Lisberger, 1992; Pastor et al., 1994; du Lac et al., 1995; Dietrich and Straka, 2016). These experimental results have inspired recent theoretical studies that suggested that memory consolidation of VOR motor learning occurs in the vestibular nuclei. Here, we provide evidence of plasticity in vestibular nuclei after a long-term reduction of the VOR. The decrease of synaptic efficacy at the level of vestibular afferent synapses onto vestibular nuclei neurons is in direct line with theoretical predictions regarding VOR gain-down adaptation (Masuda and Amari, 2008; Menzies et al., 2010; Yamazaki et al., 2015). Moreover, it is consistent with a recent study showing that a change at this synapse is sufficient to induce a persistent decrease of the VOR in vivo (Mitchell et al., 2016). This brainstem memory raises several fundamental questions, including which subpopulations of neurons are concerned and which cellular mechanisms underlie these plastic changes.

\section{The majority of MVN neurons are floccular target neurons}

MVN neurons that receive inputs from the flocculus are named floccular target neurons (FTNs). FTNs integrate cerebellar and vestibular inputs and are key players in VOR modulation. Based on in vivo studies, it was proposed that two different pathways, both projecting to ocular motoneurons, would mediate the VOR: the modifiable pathway composed of FTNs and the unmodifiable pathway composed of non-FTNs (Broussard and Kassardjian, 2004). Early in vitro electrophysiology suggested that a relatively low proportion of MVNs are FTNs $(<15 \%$; Babalian and Vidal 2000; Sekirnjak et al. 2003). Importantly, recent anatomical studies performed on mice demonstrated that the majority of MVNs are actually FTNs ( $80 \%$; Shin et al. 2011), which segregate in subpopulations according to the amount (dense vs. sparse) and 
location (somatic vs. dendritic) of cerebellar inputs received as well as their neurotransmitter content (glutamatergic, glycinergic, GABAergic; see Fig. 1; Shin et al., 2011; Matsuno et al., 2016).

Here, because FTNs were not specifically recorded, the reduction of synaptic efficacy represents the average decrease found in the entire population of second-order MVNs likely composed of densely and sparsely innervated FTNs, as well as non-FTN neurons. In line with the possibility of a widespread change in vestibular pathway, Shutoh et al. (2006) have reported an increase in the vestibular field potential, suggesting that the OKR gain-up long-term adaptation similarly concerned a majority of the vestibular neurons in vivo. Regardless of the actual proportion of MVN neurons receiving monosynaptic floccular inputs, cerebellar regulation of vestibular activity could, in the long-term, spread to non-FTNs through local networks. Shin et al. (2011) reported that about half of commissural neurons are sparsely contacted by FTNs, demonstrating the influence of cerebellar inputs on the regulation of bilateral vestibular activity beyond the first synaptic contact. This hypothesis is further considered below.

\section{Synaptic plasticity: cellular and molecular mechanisms}

The role of the input of Purkinje cells (PCs) in the induction of plasticity in the brainstem is well supported (Wulff et al., 2009; Zheng and Raman, 2010; Okamoto et al., 2011). How could PCs activity affect vestibular processing in FTNs? We demonstrated in this study that long-term VOR reduction is associated with a decrease in efficacy of the vestibular nerve synapses on MVNs, presumably through LTD-like mechanisms. It was shown that plasticity at this synapse can be induced by highfrequency stimulation of vestibular afferents, and that the direction of the plasticity is dependent on both developmental stage (Puyal et al., 2003) and stimulation pattern (Scarduzio et al., 2012) at basic potential (Idoux, 2015). Moreover, this plasticity is also dependent on the postsynaptic membrane potential (Pugh and Raman, 2006; McElvain et al., 2010). These in vitro electrophysiological data suggest that PC inhibition could guide the strengthening or weakening of vestibular nerve synapses on MVNs. The depression we report after long-term VOR reduction could be explained by a mechanism of heterosynaptic plasticity. It was theorized that this plastic process would use an anti-Hebbian input spike-timing dependent plasticity (iSTDP) mechanism, resulting from the simultaneous vestibular afferent activity and membrane hyperpolarization by PC inhibition (Menzies et al., 2010). In line with this hypothesis, a recent study demonstrated that in mouse parvocellular MVNs, inhibitory synapses from the flocculus and excitatory synapses from the vestibular nerve axons are often colocalized on distal dendrites of FTNs (Matsuno et al., 2016). Notably, the iSTDP mechanism could in theory also regulate the interaction of vestibular inputs with other, nonfloccular inhibitory inputs. In particular, the demonstration of a commissural feed-forward inhibition (Biesdorf et al. 2008;
Malinvaud et al. 2010) interleaved with cerebellar inputs (Shin et al. 2011) raises the possibility of a long-term homeostatic activity-dependent regulation of vestibular synapses strengthened by local and commissural GABAergic and glycinergic neurons (Bagnall et al., 2007; Biesdorf et al., 2008; see discussion in Menzies et al. 2010; Mitchell et al. 2016). What would be the molecular mechanisms underlying the reported synaptic plasticity? The decrease in synaptic efficacy could depend on postsynaptic alterations, with changes in the glutamatergic receptors. In support of this hypothesis, it has been demonstrated that LTD at vestibular nerve synapses on vestibular nucleus neurons depends on NMDA receptors in mice (McElvain et al., 2010; Menzies et al., 2010). Because no additional decrease can be triggered in neurons from VVM mice using standard LTD protocols, NMDA receptors are likely a major player in the plastic process. Additional work will be needed to specify the molecular mechanisms involved in this long-term synaptic plasticity.

\section{Floccular target neurons comprise both type $A$ and type B neurons}

Based on electrophysiological criteria, MVN neurons are composed of at least two subpopulations (Serafin et al., 1991). The classification used in the present study identifies type $A$ and type $B$ neurons based on the spike AHP and shape of the interspike interval (Beraneck et al., 2003). How are type $A$ and type $B$ neurons inserted into vestibular-related networks? Both types receive direct excitatory vestibular inputs (Babalian et al., 1997; Pettorossi et al., 2011) and commissural inhibition (Camp et al., 2006). The majority of type A are GABAergic neurons (Takazawa et al., 2004; Bagnall et al., 2007), which receive mostly GABAergic inhibitory inputs (Camp et al., 2006). Type $A$ neurons likely represent the majority of local interneurons (Takazawa et al., 2004) and a significant proportion of the sparse FTNs that participate in the feed-forward local regulation of MVN activity (Biesdorf et al., 2008; Malinvaud et al., 2010; Shin et al., 2011).

On the other hand, type B neurons are glutamatergic or glycinergic output neurons that project to the ocular motor nuclei (Beraneck and Idoux, 2012). Early in vitro studies reported that FTNs show membrane properties specific to a subset of type B neurons (Babalian and Vidal, 2000; Sekirnjak et al., 2003). This subpopulation of FTNs with highly nonlinear properties likely corresponds to densely innervated glycinergic neurons ( 10\%; Shin et al., 2011; Kodama et al., 2012). In addition, the majority of glutamatergic FTNs, which project axons to the ocular motor nuclei, and of glycinergic neurons, which project to the contralateral side, are also likely to be type $B$ neurons (Bagnall et al., 2007; Shin et al., 2011). Overall, available data suggest that FTNs are composed of both type A and type B neurons that are differentially inserted within vestibular networks and play functionally distinct roles.

In this study, the decrease of eEPSC amplitude was found on a population composed of both type A and type $B$ neurons: the mean eEPSC amplitude was reduced by $\sim 50 \%$ in type $A$ and $\sim 35 \%$ in type B. Although our available data do not allow for a definitive conclusion, they 
suggest that both populations are susceptible to show synaptic plasticity after VVM, and we have therefore no evidence for a differential implication of these subpopulations in the reported synaptic plasticity.

\section{Differential change in the intrinsic properties of type $A$ and type $B$ neurons}

Reorganization within the vestibular pathway has been extensively studied in the context of postlesional modifications (i.e., vestibular compensation; Curthoys, 2000; Straka et al., 2005). In addition to synaptic plasticity (Vibert et al., 2000; Grassi and Pettorossi, 2001), changes in the intrinsic membrane properties of central vestibular neurons occur over the long time scale of several weeks, with differential changes in type $A$ and type $B$ neurons (Him and Dutia, 2001; Beraneck et al., 2003, 2004). Changes in the intrinsic excitability of MVNs were already postulated as a putative mechanism after VOR learning (du Lac, 1996; Broussard and Kassardjian, 2004; Gittis and du Lac, 2006). Pettorossi et al. (2011) demonstrated on brainstem slices that high-frequency stimulation of vestibular afferents leads to differential synaptic and intrinsic plasticity in type A or type B neurons, respectively. In particular, changes in intrinsic excitability were more consistently triggered in type A neurons than in type B neurons. Here, we report a decrease in the spontaneous discharge and the intrinsic excitability of type A MVNs. Although the electrophysiological classification we use does not differentiate the heterogeneous populations of type A neurons (Kodama et al., 2012), it identifies inhibitory GABAergic neurons as a key component in the tuning of the direct vestibular pathway following long-term VOR reduction. The precise identification of the different subpopulations of MVNs using, for instance, genetically engineered mice (Bagnall et al., 2007; Kodama et al., 2012) or tracing techniques (Sekirnjak and du Lac, 2006; Matsuno et al., 2016) will be the next step in understanding the cellular mechanisms involved in VOR long-term reduction after a visual-vestibular mismatch.

\section{References}

Albus JS (1971) A theory of cerebellar function. Math Biosci 10:2561. CrossRef

Angelaki DE (2004) Eyes on target: what neurons must do for the vestibuloocular reflex during linear motion. J Neurophysiol 92:2035. CrossRef Medline

Anzai M, Kitazawa H, Nagao S (2010) Effects of reversible pharmacological shutdown of cerebellar flocculus on the memory of longterm horizontal vestibulo-ocular reflex adaptation in monkeys. Neurosci Res 68:191-198. CrossRef Medline

Babalian A, Vibert N, Assie G, Serafin M, Mühlethaler M, Vidal PP (1997) Central vestibular networks in the guinea-pig: functional characterization in the isolated whole brain in vitro. Neuroscience 81:405-426. Medline

Babalian AL, Vidal PP (2000) Floccular modulation of vestibuloocular pathways and cerebellum-related plasticity: an in vitro whole brain study. J Neurophysiol 84:2514-2528.

Bagnall MW, Stevens RJ, du Lac S (2007) Transgenic mouse lines subdivide medial vestibular nucleus neurons into discrete, neurochemically distinct populations. J Neurosci 27:2318-2330. CrossRef Medline
Baker R, Evinger C, McCrea RA (1981) Some thoughts about the three neurons in the vestibular ocular reflex. Ann N Y Acad Sci 374:171-188. Medline

Beraneck M, Idoux E (2012) Reconsidering the role of neuronal intrinsic properties and neuromodulation in vestibular homeostasis. Front Neurol 3:25. CrossRef Medline

Beraneck M, McKee JL, Aleisa M, Cullen KE (2008) Asymmetric recovery in cerebellar-deficient mice following unilateral labyrinthectomy. J Neurophysiol 100:945-958. CrossRef Medline

Beraneck M, Idoux E, Uno A, Vidal PP, Moore LE, Vibert N (2004) Unilateral labyrinthectomy modifies the membrane properties of contralesional vestibular neurons. J Neurophysiol 92:1668-1684. CrossRef Medline

Beraneck M, Hachemaoui M, Idoux E, Ris L, Uno A, Godaux E, Vidal PP, Moore LE, Vibert N (2003) Long-term plasticity of ipsilesional medial vestibular nucleus neurons after unilateral labyrinthectomy. J Neurophysiol 90:184-203. CrossRef Medline

Berthoz A, Jones GM, Bégué AE (1981) Differential visual adaptation of vertical canal-dependent vestibulo-ocular reflexes. Exp Brain Res 44:19-26. CrossRef

Biesdorf S, Malinvaud D, Reichenberger I, Pfanzelt S, Straka H (2008) Differential inhibitory control of semicircular canal nerve afferentevoked inputs in second-order vestibular neurons by glycinergic and GABAergic circuits. J Neurophysiol 99:1758-1769. CrossRef

Blazquez PM, Hirata Y, Highstein SM (2004) The vestibulo-ocular reflex as a model system for motor learning: what is the role of the cerebellum? Cerebellum 3:188-192. CrossRef Medline

Boyden ES, Raymond JL (2003) Active reversal of motor memories reveals rules governing memory encoding. Neuron 39:1031-1042. Medline

Boyden ES, Katoh A, Raymond JL (2004) Cerebellum-dependent learning: the role of multiple plasticity mechanisms. Annu Rev Neurosci 27:581-609. CrossRef Medline

Broussard DM, Lisberger SG (1992) Vestibular inputs to brain stem neurons that participate in motor learning in the primate vestibuloocular reflex. J Neurophysiol 68:1906-1909. Medline

Broussard DM, Kassardjian CD (2004) Learning in a simple motor system. Learn Mem 11:127-136. CrossRef Medline

Broussard DM, Titley HK, Antflick J, Hampson DR (2011) Motor learning in the VOR: the cerebellar component. Exp Brain Res 210:451-463. CrossRef Medline

Calabrese DR, Hullar TE (2006) Planar relationships of the semicircular canals in two strains of mice. $J$ Assoc Res Otolaryngol 7:151-159. CrossRef Medline

Camp AJ, Callister RJ, Brichta AM (2006) Inhibitory synaptic transmission differs in mouse type $A$ and $B$ medial vestibular nucleus neurons in vitro. J Neurophysiol 95:3208-3218. CrossRef

Carey MR (2011) Synaptic mechanisms of sensorimotor learning in the cerebellum. Curr Opin Neurobiol 21:609-615. CrossRef Medline

Clopath C, Badura A, De Zeeuw Cl, Brunel N (2014) A cerebellar learning model of vestibulo-ocular reflex adaptation in wild-type and mutant mice. J Neurosci 34:7203-7215. CrossRef Medline

Cullen KE (2012) The vestibular system: multimodal integration and encoding of self-motion for motor control. Trends Neurosci 35: 185-196. CrossRef Medline

Curthoys IS (2000) Vestibular compensation and substitution. Curr Opin Neurol 13:27-30. Medline

De Zeeuw Cl, Ten Brinke MM (2015) Motor learning and the cerebellum. Cold Spring Harb Perspect Biol 7:a021683. CrossRef Medline

Dietrich H, Straka H (2016) Prolonged vestibular stimulation induces homeostatic plasticity of the vestibulo-ocular reflex in larval Xenopus laevis. Eur J Neurosci 44:1787-1796. CrossRef Medline

du Lac S (1996) Candidate cellular mechanisms of vestibulo-ocular reflex plasticity. Ann N Y Acad Sci 781:489-498. Medline

du Lac S, Raymond JL, Sejnowski TJ, Lisberger SG (1995) Learning and memory in the vestibulo-ocular reflex. Annu Rev Neurosci 18:409-441. CrossRef Medline 
Dutia MB, Johnston AR, McQueen DS (1992) Tonic activity of rat medial vestibular nucleus neurones in vitro and its inhibition by GABA. Exp Brain Res 88:466-472. Medline

Dutia MB, Lotto RB, Johnston AR (1995) Post-natal development of tonic activity and membrane excitability in mouse medial vestibular nucleus neurones. Acta Otolaryngol Suppl 520 (Pt 1):101-104. CrossRef

Eugène D, Idoux E, Beraneck M, Moore LE, Vidal PP (2011) Intrinsic membrane properties of central vestibular neurons in rodents. Exp Brain Res 210:423-436. CrossRef Medline

Faulstich M, van Alphen AM, Luo C, du Lac S, De Zeeuw CI (2006) Oculomotor plasticity during vestibular compensation does not depend on cerebellar LTD. J Neurophysiol 96:1187-1195. CrossRef

Gittis AH, du Lac S (2006) Intrinsic and synaptic plasticity in the vestibular system. Curr Opin Neurobiol 16:385-390. CrossRef Medline

Grassi S, Pettorossi VE (2001) Synaptic plasticity in the medial vestibular nuclei: role of glutamate receptors and retrograde messengers in rat brainstem slices. Prog Neurobiol 64:527-553. Medline

Hansel C, Linden DJ, D’Angelo E (2001) Beyond parallel fiber LTD: the diversity of synaptic and non-synaptic plasticity in the cerebellum. Nat Neurosci 4:467-475. CrossRef Medline

Him A, Dutia MB (2001) Intrinsic excitability changes in vestibular nucleus neurons after unilateral deafferentation. Brain Res 908: 58-66. Medline

Idoux E (2015) Vestibular plasticity. In: The rat nervous system, 4th Edition (Paxinos G, ed), pp 837-842: Elsevier.

Ito $M$ (1982) Cerebellar control of the vestibulo-ocular reflex-around the flocculus hypothesis. Annu Rev Neurosci 5:275-296. CrossRef Medline

Kassardjian CD, Tan YF, Chung JY, Heskin R, Peterson MJ, Broussard DM (2005) The site of a motor memory shifts with consolidation. J Neurosci 25:7979-7985. CrossRef Medline

Keller EL, Precht W (1979) Modification of central vestibular neuron response by conflicting visual-vestibular stimulation. Prog Brain Res 50:763-770. CrossRef Medline

Kleim JA, Freeman JH, Jr., Bruneau R, Nolan BC, Cooper NR, Zook A, Walters D (2002) Synapse formation is associated with memory storage in the cerebellum. Proc Natl Acad Sci U S A 99:1322813231. CrossRef Medline

Kodama T, Guerrero S, Shin M, Moghadam S, Faulstich M, du Lac S (2012) Neuronal classification and marker gene identification via single-cell expression profiling of brainstem vestibular neurons subserving cerebellar learning. J Neurosci 32:7819-7831. CrossRef Medline

Lisberger SG (1988) The neural basis for learning of simple motor skills. Science 242:728-735. Medline

Lisberger SG, Miles FA, Optican LM (1983) Frequency-selective adaptation: evidence for channels in the vestibulo-ocular reflex? J Neurosci 3:1234-1244. Medline

Lisberger SG, Miles FA, Zee DS (1984) Signals used to compute errors in monkey vestibuloocular reflex: possible role of flocculus. J Neurophysiol 52:1140-1153. Medline

Lisberger SG, Pavelko TA, Broussard DM (1994) Neural basis for motor learning in the vestibuloocular reflex of primates. I. Changes in the responses of brain stem neurons. J Neurophysiol 72:928953. Medline

Lisberger SG, Miles FA, Optican LM, Eighmy BB (1981) Optokinetic response in monkey: underlying mechanisms and their sensitivity to long-term adaptive changes in vestibuloocular reflex. J Neurophysiol 45:869-890. Medline

Luebke AE, Robinson DA (1994) Gain changes of the cat's vestibuloocular reflex after flocculus deactivation. Exp Brain Res 98:379390. Medline

Malinvaud D, Vassias I, Reichenberger I, Rössert C, Straka H (2010) Functional organization of vestibular commissural connections in frog. J Neurosci 30:3310-3325. CrossRef Medline
Marr D (1969) A theory of cerebellar cortex. J Physiol 202:437-470. Medline

Masuda N, Amari S (2008) A computational study of synaptic mechanisms of partial memory transfer in cerebellar vestibulo-ocularreflex learning. J Comput Neurosci 24:137-156. CrossRef Medline

Matsuno H, Kudoh M, Watakabe A, Yamamori T, Shigemoto R, Nagao S (2016) Distribution and structure of synapses on medial vestibular nuclear neurons targeted by cerebellar flocculus Purkinje cells and vestibular nerve in mice: light and electron microscopy studies. PLoS One 11:e0164037. CrossRef Medline

McElvain LE, Bagnall MW, Sakatos A, du Lac S (2010) Bidirectional plasticity gated by hyperpolarization controls the gain of postsynaptic firing responses at central vestibular nerve synapses. Neuron 68:763-775. CrossRef Medline

Medina JF (2011) The multiple roles of Purkinje cells in sensori-motor calibration: to predict, teach and command. Curr Opin Neurobiol 21:616-622. CrossRef Medline

Melvill Jones G, Guitton D, Berthoz A (1988) Changing patterns of eye-head coordination during $6 \mathrm{~h}$ of optically reversed vision. Exp Brain Res 69:531-544. Medline

Menzies JR, Porrill J, Dutia M, Dean P ((2010) Synaptic plasticity in medial vestibular nucleus neurons: comparison with computational requirements of VOR adaptation. PLoS One 5. CrossRef

Miles FA, Lisberger SG (1981) Plasticity in the vestibulo-ocular reflex: a new hypothesis. Annu Rev Neurosci 4:273-299. CrossRef Medline

Mitchell DE, Della Santina CC, Cullen KE (2016) Plasticity within non-cerebellar pathways rapidly shapes motor performance in vivo. Nat Commun 7:11238. CrossRef Medline

Nagao S, Kitazawa H (2003) Effects of reversible shutdown of the monkey flocculus on the retention of adaptation of the horizontal vestibulo-ocular reflex. Neuroscience 118:563-570. Medline

Nagao S, Honda T, Yamazaki T (2013) Transfer of memory trace of cerebellum-dependent motor learning in human prism adaptation: a model study. Neural Netw 47:72-80. CrossRef Medline

Ohyama T, Mauk M (2001) Latent acquisition of timed responses in cerebellar cortex. J Neurosci 21:682-690. Medline

Ohyama T, Nores WL, Medina JF, Riusech FA, Mauk MD (2006) Learning-induced plasticity in deep cerebellar nucleus. J Neurosci 26:12656-12663. CrossRef Medline

Okamoto T, Shirao T, Shutoh F, Suzuki T, Nagao S (2011) Posttraining cerebellar cortical activity plays an important role for consolidation of memory of cerebellum-dependent motor learning. Neurosci Lett 504:53-56. CrossRef Medline

Oommen BS, Stahl JS (2008) Eye orientation during static tilts and its relationship to spontaneous head pitch in the laboratory mouse. Brain Res 1193:57-66. CrossRef Medline

Pastor AM, de la Cruz RR, Baker R (1994) Cerebellar role in adaptation of the goldfish vestibuloocular reflex. J Neurophysiol 72: 1383-1394. Medline

Pettorossi VE, Dieni CV, Scarduzio M, Grassi S (2011) Long-term potentiation of synaptic response and intrinsic excitability in neurons of the rat medial vestibular nuclei. Neuroscience 187:1-14. CrossRef Medline

Porrill J, Dean P (2007) Cerebellar motor learning: when is cortical plasticity not enough? PLoS Comput Biol 3:1935-1950. CrossRef Medline

Pugh JR, Raman IM (2006) Potentiation of mossy fiber EPSCs in the cerebellar nuclei by NMDA receptor activation followed by postinhibitory rebound current. Neuron 51:113-123. CrossRef Medline

Puyal J, Grassi S, Dieni C, Frondaroli A, Demêmes D, Raymond J, Pettorossi VE (2003) Developmental shift from long-term depression to long-term potentiation in the rat medial vestibular nuclei: role of group I metabotropic glutamate receptors. J Physiol 553: 427-443. CrossRef

Raymond JL, Lisberger SG (1996) Behavioral analysis of signals that guide learned changes in the amplitude and dynamics of the vestibulo-ocular reflex. J Neurosci 16:7791-7802. Medline

Rinaldi A, Defterali C, Mialot A, Garden DL, Beraneck M, Nolan MF (2013) HCN1 channels in cerebellar Purkinje cells promote late 
stages of learning and constrain synaptic inhibition. J Physiol 591:5691-5709. CrossRef Medline

Roy JE, Cullen KE (2004) Dissociating self-generated from passively applied head motion: neural mechanisms in the vestibular nuclei. $J$ Neurosci 24:2102-2111. CrossRef Medline

Scarduzio M, Panichi R, Pettorossi VE, Grassi S (2012) The repetition timing of high frequency afferent stimulation drives the bidirectional plasticity at central synapses in the rat medial vestibular nuclei. Neuroscience 223:1-11. CrossRef Medline

Sekirnjak C, du Lac S (2006) Physiological and anatomical properties of mouse medial vestibular nucleus neurons projecting to the oculomotor nucleus. J Neurophysiol 95:3012-3023. CrossRef Medline

Sekirnjak C, Vissel B, Bollinger J, Faulstich M, du Lac S (2003) Purkinje cell synapses target physiologically unique brainstem neurons. J Neurosci 23:6392-6398. Medline

Serafin M, de Waele C, Khateb A, Vidal PP, Mühlethaler M (1991) Medial vestibular nucleus in the guinea-pig. I. Intrinsic membrane properties in brainstem slices. Exp Brain Res 84:417-425. Medline

Shin M, Moghadam SH, Sekirnjak C, Bagnall MW, Kolkman KE, Jacobs R, Faulstich M, du Lac S (2011) Multiple types of cerebellar target neurons and their circuitry in the vestibulo-ocular reflex. $J$ Neurosci 31:10776-10786. CrossRef Medline

Shin SL, Zhao GQ, Raymond JL (2014) Signals and learning rules guiding oculomotor plasticity. J Neurosci 34:10635-10644. CrossRef Medline

Shutoh F, Ohki M, Kitazawa H, Itohara S, Nagao S (2006) Memory trace of motor learning shifts transsynaptically from cerebellar cortex to nuclei for consolidation. Neuroscience 139:767-777. CrossRef Medline
Stahl JS (2004) Using eye movements to assess brain function in mice. Vision Res 44:3401-3410. CrossRef Medline

Straka H, Lambert FM, Pfanzelt S, Beraneck M (2009) Vestibuloocular signal transformation in frequency-tuned channels. Ann N Y Acad Sci 1164:37-44. CrossRef Medline

Straka H, Vibert N, Vidal PP, Moore LE, Dutia MB (2005) Intrinsic membrane properties of vertebrate vestibular neurons: function, development and plasticity. Prog Neurobiol 76:349-392. CrossRef Medline

Takazawa T, Saito Y, Tsuzuki K, Ozawa S (2004) Membrane and firing properties of glutamatergic and GABAergic neurons in the rat medial vestibular nucleus. J Neurophysiol 92:3106-3120. CrossRef Medline

van Alphen B, Winkelman BH, Frens MA (2010) Three-dimensional optokinetic eye movements in the C57BL/6J mouse. Invest Ophthalmol Vis Sci 51:623-630. CrossRef Medline

Vibert N, Beraneck M, Bantikyan A, Vidal PP (2000) Vestibular compensation modifies the sensitivity of vestibular neurones to inhibitory amino acids. Neuroreport 11:1921-1927. Medline

Wulff $P$, Schonewille $M$, Renzi $M$, Viltono L, Sassoè-Pognetto $M$, Badura A, Gao Z, Hoebeek FE, van Dorp S, Wisden W, Farrant M, De Zeeuw Cl (2009) Synaptic inhibition of Purkinje cells mediates consolidation of vestibulo-cerebellar motor learning. Nat Neurosci 12:1042-1049. CrossRef Medline

Yamazaki T, Nagao S, Lennon W, Tanaka S (2015) Modeling memory consolidation during posttraining periods in cerebellovestibular learning. Proc Natl Acad Sci U S A 112:3541-3546. CrossRef Medline

Zheng N, Raman IM (2010) Synaptic inhibition, excitation, and plasticity in neurons of the cerebellar nuclei. Cerebellum 9:56-66. CrossRef Medline 